\title{
Sigmund Freud
}

\section{ESQUISSE / ENTWURF}

(Document de travail : traduction Suzanne Hommel, avec la participation de André Albert, Éric Laurent, Guy Le Gauffey, Erik Porge), Extrait de Palea 6,7 et 8. 


\section{Plan Général \\ INTRODUCTION}

Le projet de cette esquisse est d'aboutir à une psychologie comme science de la nature c'est-à-dire représentant les processus psychiques comme des états quantitativement déterminés de particules matérielles distinguables, ceci afin de les rendre figurables et non contradictoires. L'esquisse contient deux idées principales :

1) comprendre ce qui distingue l'activité du repos comme une quantité (Q) soumise aux lois générales du mouvement,

2) supposer que les neurones sont des particules matérielles $N$ et $Q \eta$-Des tentatives analogues sont maintenant fréquentes.

\section{PREMIER PRINCIPE}

\section{1 - LA CONCEPTION QUANTITATIVE}

Elle est directement tirée des observations cliniques de la pathologie, surtout là où il s'agissait de représentations hyperintenses, comme dans l'hystérie et la névrose obsessionnelle, où, comme cela s'avérera, le caractère quantitatif ressort plus nettement que dans le normal. Des processus comme la stimulation, la substitution, la conversion, la décharge, qui étaient à décrire, ont directement suggéré la conception de l'excitation neuronique en termes d'écoulements de quantités. II semblait possible de généraliser ce qui est ici admis. On pourrait poser à partir de ces considérations le principe fondamental d'activité des neurones en rapport avec la quantité (Q), principe qui promettait d'éclairer la question puisqu'il semblait embrasser l'ensemble de la fonction. C'est le principe de l'inertie des neurones ; il énonce que les neurones tendent à se défaire de la quantité. La structure et le développement ainsi que le travail des neurones, sont à comprendre selon ce principe.

Le principe d'inertie explique d'abord la structure clivée des neurones en neurones moteurs et neurones sensitifs comme étant un dispositif destiné à annuler (aufheben) l'absorption des quantités en les transmettant. On peut ainsi comprendre le mouvement réflexe comme forme figée de cette transmission de quantités. Le principe d'inertie fournit le motif du mouvement réflexe. Si nous remontons plus loin, nous avons le système neuronique qui, en tant qu'héritier de l'irritabilité générale du protoplasme, se trouve d'abord à la surface extérieure irritable qui est morcelée par de larges bandes de ... non-excitables. Un système neuronique primaire se sert de cette quantité $(Q \eta)$ ainsi acquise afin de la transmettre, grâce à l'existence d'une liaison, aux machines musculaires, et se maintient ainsi en non-stimulation (reizlos). Cette décharge figure la fonction primaire des systèmes neuroniques. II y a place maintenant pour le développement d'une fonction secondaire : la fuite devant les stimulations. En effet, parmi les voies de décharge, sont préférées et maintenues celles auxquelles la cessation de la stimulation est liée. II y a ici en général une proportion qui s'établit entre la quantité d'excitation et le travail nécessaire à la fuite devant la stimulation, afin que le principe d'inertie ne soit pas perturbé de ce fait. Mais, dés le départ, le principe d'inertie est perturbé par d'autres facteurs. Quand la complexité s'accroît à l'intérieur, le système neuronique reçoit des stimulations venant de l'élément corporel lui-même, des stimulations endogènes qui doivent aussi être déchargées Celles-ci trouvent leur origine dans les cellules du corps et il en résulte les 3 grands besoins -la faim, la respiration, la sexualité-. L'organisme ne peut 
échapper à ces grands besoins comme il peut échapper aux stimulations venues de l'extérieur, il ne peut pas utiliser leur quantité pour fuir la stimulation. Ces besoins ne cessent que dans des conditions déterminées qui doivent nécessairement être réalisées dans le monde extérieur. Par exemple, le besoin de nourriture. Afin d'accomplir cette action qui mérite d'être appelée spécifique, il faut un travail qui est indépendant des quantités $(Q \eta)$ et qui est généralement plus grand, puisque l'individu est placé dans des conditions qu'on peut désigner comme constituant la Nécessite de la vie (Not des Lebens). Par là, le système neuronique est forcé d'abandonner la tendance originaire à l'inertie, c'est-à-dire à la réduction à un niveau $=0$. II faut qu'il apprenne à supporter une réserve de quantité pour satisfaire aux exigences d'une action spécifique. Dans la façon dont il le fait, apparaît néanmoins la persistance de la même tendance sous la forme modifiée d'un effort pour maintenir au moins au niveau le plus bas possible la quantité $(Q \eta)$, et pour se défendre contre une augmentation de celle-ci, c'est-à-dire pour la maintenir constante. Tout le travail du système neuronique est à considérer soit du point de vue de la fonction primaire, soit de celui de la fonction secondaire qui est imposée par la Nécessité de la vie.

\section{2 - LA THEORIE DES NEURONES}

Le deuxième pilier de cette doctrine est de combiner cette théorie de la quantité $(\mathrm{Q} \eta)$ avec ce que nous savons des neurones d'après la plus récente histologie. Son apport principal est que le système neuronique est constitué de neurones distincts, de structure analogue, en contact par l'intermédiaire d'une masse étrangère. De l'un à l'autre, les extrémités se comportent comme des parties de tissu étranger dans lesquelles certaines directions de conduction sont préformées ; en effet, ils reçoivent au moyen d'appendices cellulaires et transmettent par des cylindres-axes. A cela s'ajoutent de nombreuses ramifications de calibre très différent.

Si l'on combine cette présentation des neurones avec notre conception de la théorie des quantités $(\mathrm{Q} \eta)$, on obtient la représentation d'un neurone $\mathrm{N}$ investi rempli d'une certaine quantité $(Q \eta)$, qui à d'autres moments peut être vide. Le principe d'inertie trouve son expression dans l'hypothèse d'un courant dirigé, à partir des voies de conduction ou appendices cellulaires, vers le cylindre-axe. Chaque neurone est ainsi à l'image du système neuronique tout entier avec sa structure clivée, le cylindre-axe étant l'organe de décharge. Toutefois la fonction secondaire qui exige un emmagasinement de quantité $(\mathrm{Q} \eta)$ est rendue possible si l'on suppose des résistances qui s'opposent à la décharge, et la structure des neurones permet de penser que toutes les résistances se produisent aux points de contact qui prennent ainsi la valeur de barrières. L'hypothèse de barrières de contact est féconde à bien des égards.

\section{3 - LES BARRIERES DE CONTACT}

La première justification de cette hypothèse correspond à ce que la conduction passe par un protoplasme indifférencié au lieu de passer, comme ailleurs, à l'intérieur du neurone, par un protoplasme différencié, vraisemblablement plus propre à la conduction. On obtient ainsi une indication permettant de rattacher la conductibilité à la différenciation. On est ainsi en droit de s'attendre à ce que, par le processus de conduction lui-même, soit créée une différenciation dans le protoplasme, et par là une meilleure conductibilité pour des conductions ultérieures.

En outre, la théorie des barrières de contact autorise d'autres usages. Une propriété essentielle du tissu nerveux est la mémoire, c'est-à-dire de façon tout à fait 
générale l'aptitude à être modifié de façon permanente par des processus qui n'ont lieu qu'une fois, ce qui contraste étonnamment avec le comportement d'une matière qui laisserait passer un mouvement ondulatoire, et qui retournerait ensuite à son état antérieur. Une théorie psychologique digne de quelque attention doit fournir une explication de la "mémoire". Mais une telle explication se heurte toujours à la difficulté suivante. D'un côté elle doit supposer que les neurones sont modifiés après leur excitation, de façon permanente par rapport à ce qu'ils étaient auparavant. De l'autre, pourtant, on ne peut nier que les nouvelles excitations rencontrent en général les mêmes conditions de réception que les excitations antérieures. Les neurones doivent donc être à la fois influencés et inchangés, sans parti pris. A première vue, nous ne pouvons imaginer un appareil qui puisse accomplir ce travail complexe. La solution consiste à attribuer la modification permanente résultant de l'excitation à une classe de neurones, et l'inaltérabilité, donc la fraîcheur pour de nouvelles excitations, à une autre. D'où la distinction opératoire entre "cellules de perception" et "cellules de souvenir", bien qu'elle ne s'insère dans rien d'autre et qu'elle ne repose sur rien de connu.

Si l'on tient compte maintenant de la théorie des barrières de contact, on en vient à reformuler les choses ainsi. II y a deux classes de neurones. Premièrement ceux qui laissent passer la quantité $(\mathrm{Q} \eta)$ comme s'ils n'avaient pas de barrières de contact, qui sont donc après chaque écoulement d'excitation dans le même état qu'auparavant ; et deuxièmement ceux dont les barrières de contact ont pour fonction de ne laisser passer de la quantité $(Q \eta)$ que difficilement ou partiellement. Ces derniers peuvent se trouver après chaque excitation dans un autre état qu'auparavant fournissant donc une possibilité de représenter la mémoire.

II y a donc des neurones perméables (qui n'exercent aucune résistance et qui ne retiennent rien) qui servent à la perception, et des neurones imperméables (ayant une résistance et retenant de la quantité $(Q \eta)$ ) qui sont le support de la mémoire, donc probablement des processus psychiques en général. Je nommerai donc, à partir de maintenant, le premier système de neurones, le second $\Psi$.

II faudrait maintenant préciser quelles hypothèses concernant les neurones sont nécessaires pour rendre compte des caractères les plus généraux de la mémoire. L'argument est le suivant : ils sont modifiés de façon durable par le cours de l'excitation. En faisant intervenir la théorie des barrières de contact : leurs barrières de contact se trouvent donc dans un état durablement modifié. Et puisque l'expérience psychologique montre qu'il y a un sur-apprentissage dû à la mémoire, cette modification doit nécessairement consister en ceci que les barrières de contact deviennent plus aptes à la conduction, moins imperméables, donc plus semblables à celles du système $\phi$. Nous désignerons cet état des barrières de contact comme étant le degré du frayage (Bahnung). On peut dire alors : la mémoire est représentée par les frayages existant entre les neurones $\Psi$.

Si nous supposions que toutes les barrières de contact $\Psi$ étaient aussi bien frayées les unes que les autres, ou encore qu'elles offraient la même résistance, ce qui est la même chose, les caractères de la mémoire ne ressortiraient évidemment pas. Car la mémoire est bien évidemment, par rapport à l'écoulement de l'excitation, une des puissances déterminantes qui indiquent la voie à suivre, et dans le cas d'un frayage partout identique, on ne verrait pas comment il y aurait choix entre différentes voies. D'où l'on peut dire d'une manière encore plus exacte : la mémoire serait représentée (dargestellt) par les différences de frayage entre les neurones $\Psi$. 
De quoi dépend donc le frayage dans les neurones $\Psi$ ? Selon l'expérience psychologique, la mémoire, à savoir la force qui continue à travailler après un événement, dépend d'un facteur qu'on appelle l'intensité de l'impression, et de la fréquence de la répétition de cette même impression. Ce qui se traduit dans la théorie par : le frayage dépend de la quantité $(Q \eta)$ qui passe à travers le neurone au cours du processus d'excitation, ainsi que du nombre de répétitions du processus. Ainsi la quantité $(Q \eta)$ s'avère être le facteur qui travaille, la quantité et le frayage apparaissent comme l'effet de la quantité $(Q \eta)$, en même temps comme ce qui peut remplacer la quantité.

On ne peut pas ne pas penser ici à l'effort premier des systèmes neuroniques qui persistent à travers toutes les modifications, pour s'épargner la surcharge par la quantité $(Q \eta)$ ou pour la diminuer le plus possible. II a fallu que le système neuronique fasse provision d'une réserve de quantité (Qn), forcé qu'il était par la nécessité de la vie. Pour cela, il a eu besoin d'une multiplication du nombre de ses neurones, et qu'ils soient imperméables. Dans le même temps, il s'épargne, dans une certaine mesure tout au moins, le comblement par la quantité ( $Q \eta)$, l'investissement, et ce en établissant les frayages. On le voit donc, les frayages servent la fonction primaire.

L'application de ce qu'exige la mémoire sur la théorie des barrières de contact exige autre chose encore : en général à chaque neurone peuvent être attribuées plusieurs voies de liaison avec d'autres neurones, donc plusieurs barrières de contact. C'est bien là-dessus que repose la possibilité du choix qui est déterminé par le frayage. II est maintenant tout à fait clair qu'il faut que l'état de frayage d'une des barrières de contact soit indépendant de celui de toutes les autres barrières de contact des mêmes neurones $\Psi$; sinon, de nouveau on n'aurait aucune préférence, donc aucun motif. De ceci, on peut tirer une conclusion négative concernant la nature de l'état "frayé". Si l'on s'imagine un neurone comblé de quantité $(Q \eta)$, donc investi, on ne peut supposer cette quantité $(Q)$ que régulièrement répartie sur toutes les régions du neurone, donc aussi sur toutes les barrières de contact de celui-ci. Par

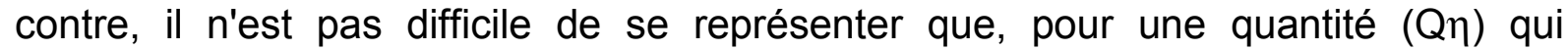
s'écoule, seule une voie déterminée est empruntée à travers le neurone, de façon à ce qu'une seule barrière de contact soit soumise au travail de la quantité $(Q \eta)$ qui s'écoule et en conserve par la suite du frayage. Le frayage ne peut donc avoir son fondement dans un investissement en rétention qui ne pourrait donner de différences de frayage entre les barrières de contact du même neurone.

La question de savoir en quoi consiste le frayage reste par ailleurs en suspens. On pourrait penser tout d'abord : dans l'absorption de quantité $(\mathrm{Q} \eta)$ par les barrières de contact. Peut-être la lumière se fera-t-elle ultérieurement là-dessus. La quantité $(Q \eta)$ qui a laissé après elle le frayage est bien déchargée, précisément en raison du frayage qui, comme nous le savons, augmente la perméabilité. II n'est par ailleurs pas nécessaire que le frayage qui subsiste après un écoulement de quantité $(Q \eta)$ soit aussi grand qu'il devait l'être pendant l'écoulement. Possible qu'il n'en reste comme frayage durable qu'une fraction (Quotientbetrag). Dans cette mesure, on ne peut pas encore voir s'il y a équivalence entre l'écoulement d'une quantité $3(Q \eta)$ en une fois et l'écoulement d'une quantité $(Q \eta)$ en trois fois. Tout ceci est formulé sous réserve d'adaptations ultérieures de la théorie aux faits psychologiques. 


\section{4 - LE POINT DE VUE BIOLOGIQUE}

Si l'on suppose deux systèmes neuroniques $\phi$ et $\Psi$, dont le premier est constitué par des éléments perméables et le second par des éléments imperméables, une particularité du système neuronique, sa capacité de retenir et de demeurer quand même réceptif, semble s'expliquer. Toute acquisition psychique reposerait dès lors sur une organisation du système $\Psi$ caractérisée par la levée (Aufhebung) partielle et topiquement déterminée de la résistance dans les barrières de contact qui différencie $\phi$ et. Avec le développement de celle-ci, la capacité du système neuronique de recevoir trouverait en fait une limite.

Néanmoins, tous ceux qui construisent scientifiquement des hypothèses scientifiques ne commencent à les prendre au sérieux que lorsqu'elles s'intègrent de plus d'une façon dans le savoir établi et lorsque l'arbitraire de la construction ad hoc s'y trouve tempéré. On objectera à notre hypothèse des barrières de contact qu'elle suppose deux classes de neurones avec une différenciation fondamentale quant aux conditions de fonctionnement et que, pour l'instant, toute espèce de fondement fait défaut pour soutenir cette séparation. Du moins elle ne s'appuie sur rien de morphologique, c'est-à-dire d'histologique.

A partir de quoi doit-on alors fonder cette division en classes ? Si possible à partir du développement biologique du système neuronique qui, pour le naturaliste, est, comme tout autre système, le résultat d'une évolution progressive. II faudrait savoir si les deux classes de neurones pourraient avoir eu une signification biologiquement différente, et, dans ce cas, par quel mécanisme elles ont bien pu se développer jusqu'à présenter des caractéristiques aussi différentes que celles de perméabilité et d'imperméabilité. Naturellement, la solution la plus satisfaisante serait que le mécanisme recherché puisse résulter lui-même de leur rôle biologique primitif ; on aurait alors résolu deux questions avec une seule réponse.

Souvenons-nous maintenant que le système neuronique avait dés le début deux fonctions : accueillir les stimulations venues de l'extérieur et décharger les excitations d'origine endogène. C'est de cette dernière obligation que résulta, du fait de la nécessité de la vie, la contrainte à un développement biologique ultérieur. On pourrait dès lors supposer que ce sont précisément nos systèmes $\phi$ et $\Psi$ qui se sont répartis sur ces obligations primaires. Le système $\phi$ serait le groupe de neurones où aboutissent les stimulations extérieures ; le système $\Psi$ contiendrait les neurones qui reçoivent les excitations endogènes. Ainsi nous n'aurions pas inventé mais au contraire trouvé les deux systèmes $\phi$ et $\Psi$. II reste encore à les identifier à quelque chose de connu. De fait, nous connaissons par l'anatomie un système de neurones (la substance grise de la moelle) qui seul est en connexion avec le monde extérieur, et par ailleurs un système superposé (la substance grise du cerveau) qui n'a pas de liaisons périphériques directes, mais auquel se relient le développement du système neuronique et les fonctions psychiques. Le cerveau primaire s'accorde assez bien avec notre description du système $\Psi$ si toutefois il nous est permis de supposer que le cerveau a des voies directes et indépendantes de $\phi$ vers l'intérieur du corps. La provenance et la signification biologique originaire du cerveau primaire ne sont, il est vrai, pas connues des anatomistes. Selon notre théorie, ce serait, pour le dire sans détours, un ganglion du sympathique. C'est ici la première possibilité qui s'offre de mettre la théorie à l'épreuve à l'aide d'un matériel réel. 
Identifions provisoirement le système $\Psi$ à la substance grise du cerveau. On comprend bien alors à partir des remarques biologiques qui précèdent, que c'est précisément $\Psi$ qui est sujet à l'évolution due à la multiplication des neurones et à l'accumulation de quantité ; on voit aussi combien il est utile que $\Psi$ soit constitué de neurones imperméables, puisque sans cela il serait incapable de faire face aux exigences de l'action spécifique. Mais par quelle voie $\Psi$ a-t-il acquis cette propriété d'imperméabilité ?

$\Phi$ a pourtant, lui aussi, des barrières de contact, mais si elles ne jouent aucun rôle, pourquoi celles de $\Psi$ en jouent-elles un ? L'hypothèse d'une différenciation première dans la valeur des barrières de contact de $\phi$ et de $\Psi$ présente encore une fois un caractère fâcheusement arbitraire, bien que maintenant, selon le raisonnement de Darwin, on puisse invoquer le fait que, des neurones imperméables étant absolument indispensables, cela implique qu'ils survivent.

Un autre moyen de s'en sortir semble être plus fécond et plus modeste. Rappelons-nous que les barrières de contact des neurones $\Psi$ sont finalement, elles aussi, soumises au frayage et que c'est la quantité $(Q \eta)$ qui les fraie. Plus la quantité dans l'écoulement de l'excitation est grande, plus augmente le frayage, et donc plus ils se rapprochent des caractères des neurones $\phi$. Ainsi attribuons-nous les différences, non pas aux neurones, mais aux quantités auxquelles elles ont affaire. On peut donc présumer que sur les neurones se déversent des quantités pour lesquelles la résistance des barrières de contact n'entre pas en ligne de compte, alors que n'aboutissent aux neurones $\Psi$ que des quantités qui sont de l'ordre de grandeur de cette résistance. Ainsi un neurone $\phi$ deviendrait imperméable et un neurone $\Psi$ perméable, si nous pouvions permuter (Verbindung -nouer, joindre, raboutir- vertauschen : échanger-) leur topique et leurs connexions. Ils conservent pourtant leurs caractéristiques parce qu'ils ne sont en connexion, pour l'un, le neurone $\phi$, qu'avec la périphérie, pour l'autre, le neurone $\Psi$, qu'avec l'intérieur du corps. La différence de nature est remplacée par une différence de milieu et de destin.

Mais maintenant nous avons à examiner l'hypothèse selon laquelle les quantités de stimulation atteignant les neurones depuis la périphérie extérieure sont d'un ordre supérieur à celles venant de la périphérie intérieure du corps ; Bien des arguments plaident effectivement pour cette hypothèse.

Tout d'abord, il est indubitable que le monde extérieur est à l'origine de toutes les grandes quantités d'énergie, puisque, selon nos connaissances physiques, il est constitué par des masses puissantes, violemment mues, qui propagent leur mouvement. Le système $\phi$ qui est tourné vers le monde extérieur aura la tâche de décharger le plus rapidement possible les quantités $(Q \eta)$ envahissant les neurones ; il sera pourtant de toutes façons exposé à l'influence de grandes quantités $(Q)$.

Le système $\Psi$, d'après ce que nous savons, est exclu de toute liaison avec le monde extérieur ; il ne reçoit de quantités (Q) que, d'une part, des neurones $\phi$ euxmêmes, d'autre part, des éléments cellulaires à l'intérieur du corps, et il s'agit maintenant d'établir que ces quantités de stimulation sont vraisemblablement d'un ordre de grandeur inférieur. II peut sembler gênant au premier abord de devoir reconnaître aux neurones $\Psi$ deux sources de stimulation aussi différentes que $\phi$ et les cellules de l'intérieur du corps; mais c'est précisément ici que l'histologie récente des systèmes neuroniques nous apporte une aide décisive. Elle montre que les 
terminaisons des neurones et leurs connexions sont construites sur le même modèle, que les neurones sont liés les uns aux autres (aneinander endingen), comme ils le sont aux éléments somatiques. Les deux processus ont probablement le même rôle fonctionnel. II s'agira vraisemblablement pour la terminaison nerveuse de quantités similaires à celles de la conduction intercellulaire. Nous pouvons aussi nous attendre à ce que les stimulations endogènes appartiennent à cet ordre de grandeur intercellulaire. S'ouvre ici un deuxième accès à la vérification de la théorie.

\section{5 - LE PROBLEME DE LA QUANTITE}

Je ne sais rien de la grandeur absolue des stimulations intercellulaires, mais je me permettrais de faire l'hypothèse qu'étant d'un ordre de grandeur moindre, elles sont du même ordre de grandeur que celui des résistances des barrières de contact. S'il en est ainsi, on comprend facilement. Avec cette supposition, l'identité des neurones $\phi$ et $\Psi$ est sauvée et leur différenciation en ce qui concerne la perméabilité est expliquée biologiquement et mécaniquement

Ici nous manquons de preuves, d'où l'intérêt d'autant plus grand de certaines indications et conceptions qui se rattachent à notre hypothèse. Tout d'abord, lorsqu'on se sera fait une idée exacte de la grandeur des quantités $(Q)$ dans le monde extérieur, on se demandera si la tendance primaire du système neuronique à maintenir la quantité $(Q \eta)$ à zéro ne trouve pas à se satisfaire par une décharge rapide, et si elle n'est pas déjà à l'oeuvre lors de la réception des stimulations. En effet, on voit les neurones $\phi$ se terminer non pas librement à la périphérie, mais au contraire dans des structures cellulaires qui reçoivent à leur place la stimulation exogène. Ces "appareils nerveux terminaux", au sens le plus général, pourraient bien avoir pour but de ne pas laisser agir sur $\phi$ les quantités $(Q)$ sans diminution de leur intensité, mais de les amortir. Ils auraient dès lors le sens d'écrans protecteurs (Quantitätsschirmen: protection contre la quantité (Q), écrans(?) à travers lesquels ne passent que des quotients (Quotienten) des quantités exogènes $(Q)$.

Ceci concorde avec le fait que l'autre sorte de terminaison nerveuse -celle qui est libre et sans organes terminaux, à la périphérie interne du corps- est de loin la plus courante. Nul écran protecteur(Quantitätsschirme) ne parait ici nécessaire à l'encontre des quantités $(Q)$, probablement parce que les quantités $(Q \eta)$ pouvant être reçues ici n'exigent pas d'abord d'être ramenées à l'ordre de grandeur intercellulaire mais au contraire sont de prime abord telles.

Puisqu'on peut calculer les quantités (Q) qui sont reçues par les terminaisons nerveuses des neurones $\phi$, on a peut-être ici le moyen de se donner une représentation des grandeurs qui s'écoulent entre les neurones $\Psi$ et qui sont donc du même ordre que les résistances des barrières de contact.

On pressent ici en outre l'existence d'une tendance qui pourrait bien déterminer la structure du système neuronique en tant que composé de plusieurs systèmes : tendance à écarter toujours plus la quantité $(Q \eta)$ des neurones. Ainsi la structure du système neuronique pourrait servir à tenir à l'écart des neurones la quantité $(Q \eta)$, tandis que sa fonction pourrait être de les en décharger. 


\section{6 - LA DOULEUR}

Tous les dispositifs de nature biologique ont leurs limites d'efficacité en dehors desquelles ils refusent (Versagen: refuser...) de fonctionner. Ce refus (Dies Versagen: ces défaillances, ces ratés) s'exprime dans des phénomènes frisant le pathologique, qui fournissent pour ainsi dire les modèles servant de normes pour le pathologique. Nous avons découvert dans le système neuronique une organisation telle que les grandes quantités extérieures $(Q)$ sont maintenues à l'écart de $\phi$ et plus encore de $\Psi$; les écrans des terminaisons nerveuses et la liaison purement indirecte de $\Psi$ avec le monde extérieur servent ce but. $Y$ a-t-il un phénomène que l'on puisse faire correspondre au refus (Versagen) de fonctionner de ces dispositifs ? Je crois que c'est la douleur.

Tout ce que nous savons de la douleur s'y accorde. Le système neuronique a la tendance la plus radicale à la fuite devant la douleur. Nous y voyons l'expression de la tendance primaire à éviter l'augmentation de la tension de quantité ( $Q \eta)$, et nous en concluons que la douleur consiste dans l'irruption de grandes quantités (Q) dans $\Psi$. Dès lors les deux tendances n'en constituent plus qu'une seule et unique. La douleur met en mouvement les systèmes $\phi$ et $\Psi$, sa conduction ne rencontre aucun obstacle, elle est le plus impérieux de tous les processus. Les neurones semblent donc lui être perméables, elle consiste dans l'action de quantités ( $Q$ ) d'un ordre supérieur.

La douleur peut résulter d'une part d'une augmentation de quantité; toute excitation sensorielle tend à devenir de la douleur avec l'accroissement de la stimulation, et ceci même lorsqu'il s'agit des organes sensoriels supérieurs. Ceci est à comprendre tout simplement comme un refus (versagen) de fonctionner. D'autre part, s'il y a de la douleur là où les quantités extérieures sont faibles, elle est alors régulièrement liée à une solution de continuité, c'est-à-dire des quantités extérieures (Q) qui agissent directement sur les terminaison. des neurones $\phi$, et non pas à travers les appareils nerveux terminaux, donnent de la douleur. Ainsi la douleur estelle caractérisée par l'irruption de quantités excessives (Q) dans $\phi$ et $\Psi$, c'est-à-dire de quantités ( $Q$ ) dont l'ordre de grandeur est encore plus élevé que celui des stimulations $\phi$.

Que la douleur emprunte toutes les voies de décharge est facile à comprendre. D'après notre théorie, selon laquelle la quantité $(Q)$ produit du frayage, la douleur laisse bien derrière elle en $\Psi$ des frayages permanents, comme si la foudre était tombée, frayages qui peuvent supprimer complètement la résistance des barrières de contact et y établir une voie de conduction telle qu'il en existe en $\phi$.

\section{7 - LE PROBLEME DE LA QUALITE}

Toute théorie psychologique -cela n'a pas été dit jusqu'à présent- doit nécessairement, en plus du programme qui est le sien en tant que science de la nature, satisfaire à une autre exigence importante. Elle doit nous expliquer ce que nous connaissons de la façon la plus énigmatique par notre "conscience", et, puisque cette conscience ne sait rien des suppositions faites jusqu'à présent -quantités et neurones- cette théorie doit aussi nous expliquer ce non-savoir. 
Nous commençons maintenant à comprendre une hypothèse qui nous a conduit jusqu'à présent. Nous avons traité les processus psychiques comme quelque chose qui pourrait se passer de cette connaissance par la conscience, quelque chose qui existe indépendamment de celle-ci. Nous nous attendons à ne pas trouver confirmées par la conscience quelques-unes de nos hypothèses. Si nous ne nous laissons pas dérouter par cela, c'est parce que nous supposons que la conscience ne fournit une connaissance ni complète ni fiable des processus neuroniques. Ceuxci, envisagés dans toute leur étendue, doivent être considérés avant tout comme inconscients et ils doivent être inférés comme d'autres choses de la nature.

Le contenu de la conscience est alors à ranger parmi nos processus quantitatifs $\Psi$. La conscience nous fournit ce que nous appelons des qualités, des sensations qui sont autres en présentant une grande multiplicité de différences et dont l'altérité est distinguée suivant les relations au monde extérieur. Dans cet autre il y a des séries, des analogies, etc., mais point de quantités à proprement parler. On peut se demander comment se forment les qualités et où elles se constituent. Ce sont là des questions nécessitant un examen des plus attentifs, mais qui ne peuvent être traitées ici qu'approximativement.

Où les qualités se forment-elles: pas dans le monde extérieur, car, d'après notre conception des sciences de la nature sur laquelle la psychologie elle aussi doit se régler, il n'y a à l'extérieur que des masses en mouvement et rien d'autre. Serait ce dans le système $\phi$ ? Ceci s'accorde avec le fait que les qualités sont à la perception, mais est contredit par tous les arguments légitimes situant le siège de la conscience dans les étages supérieurs du système neuronique. C'est donc dans le système $\Psi$. Cependant une objection importante va à l'encontre de cela. Lors de la perception, les systèmes $\phi$ et $\Psi$ ont une activité solidaire; et ce, alors que s'effectue un processus psychique unique qui s'accomplit bien exclusivement en $\Psi$, la reproduction ou remémoration: ce processus est, pour le dire en termes généraux, sans qualité. Normalement la remémoration ne fait rien apparaître de la particularité propre à la qualité de la perception. Ainsi trouve-t-on le courage de faire l'hypothèse de l'existence d'un troisième système de neurones -neurones de perception en quelque sorte- lequel est coexcité lors de la perception, mais non lors de la reproduction, et dont les états d'excitation fournissent les différentes qualités, c'est-àdire des sensations conscientes.

Si l'on maintient fermement que notre conscience ne livre que des qualités, alors que les sciences de la nature ont affaire à des quantités, il en résulte comme par une règle de trois une caractéristique des neurones de perception. Tandis que la science s'est en effet donnée pour tâche de ramener toutes les qualités constitutives de la sensation à la quantité extérieure, la structure du système neuronique laisse présager que ce troisième système consiste en dispositifs destinés à transformer la quantité extérieure en qualité, en quoi la tendance originaire à tenir à l'écart la quantité apparaît de nouveau victorieuse. Les appareils formés par les terminaisons nerveuses constituaient un écran, afin de ne laisser agir sur $\phi$ que des quotients de la quantité extérieure, cependant que $\phi$ accomplit simultanément le gros de la décharge de la quantité. Le système $\Psi$ était déjà protégé contre des quantités d'ordre supérieur, il n'avait affaire qu'à des quantités intercellulaires Si l'on poursuit, il faut supposer que le système $P$ est mû par des quantités encore moindres. On devine que le caractère distinctif de la qualité (donc la sensation consciente) n'apparaît que là où les quantités sont dans toute la mesure du possible mises hors 
circuit. Elles ne sont pas susceptibles d'être entièrement éliminées, car nous devons penser ces neurones de perception comme investis de quantité $(\mathrm{Q} \eta)$ et s'efforçant de la décharger.

Mais c'est alors que surgit une difficulté apparemment énorme. Nous avons vu que la perméabilité dépend de l'action de la quantité $(Q \eta)$, et que les neurones $\Psi$ sont déjà imperméables. Alors qu'il y a passage d'une quantité $(\mathrm{Q} \eta)$ encore plus petit, les neurones de perception devraient être encore plus imperméables. Mais nous ne pouvons pas attribuer ce caractère aux neurones-supports de la conscience. La variation de leurs contenus, la fugacité de la conscience, le nouage aisé de qualités simultanément perçues, tout cela ne peut s'accorder qu'avec une complète perméabilité des neurones de perception accompagnée d'une totale restitution in integrum. Les neurones de perception se comportent comme des organes de perception, et nous ne saurions que faire, en ce qui les concerne, d'une mémoire. Donc la perméabilité alliée à un frayage complet qui ne provient pas de quantités. Mais alors d'où résulte-t-il?

Je ne vois qu'une issue: réviser l'hypothèse fondamentale sur l'écoulement de la quantité $(\mathrm{Q} \eta)$. Jusqu'ici je ne considère celui-ci que comme un transfert de quantité $(Q \eta)$ d'un neurone à l'autre. Mais il faut encore qu'il présente un autre caractère, de nature temporelle, car la mécanique des physiciens a laissé également aux autres mouvements de masse du monde extérieur cette caractéristique temporelle. Je l'appelle en un mot la période. Je supposerai donc que toute résistance des barrières de contact ne s'applique qu'au transfert de quantité $(\mathbf{Q})$, mais que la période du mouvement neuronique se propage dans toutes les directions sans être tombée, comme s'il s'agissait d'un processus d'induction.

L'explication physique a ici encore beaucoup à faire car là aussi les lois générales du mouvement doivent s'appliquer sans contradiction. Mon hypothèse va encore plus loin: les neurones de perception, incapables d'assurer la réception des quantités $(Q \eta)$, assimilent en revanche la période de l'excitation, et cet état au cours duquel ils sont affectés par une période moyennant un comblement minime par la quantité, $\{Q \eta)$ constitue le fondement de la conscience. Les neurones $\phi$, eux aussi, ont naturellement leur période, mais celle-ci est dépourvue de qualité, pour mieux dire : monotone. Les écarts par rapport à cette période psychique propre surgissent sous forme de qualités dans la conscience.

D'où proviennent les différences de période ? Des organes des sens, tout l'indique. Leurs qualités doivent être représentées par différentes périodes du mouvement neuronique. Les organes sensoriels agissent non seulement comme des écrans contre la quantité $(Q)$ de même que tous les appareils de terminaison nerveuse, mais aussi comme des tamis: en ne laissant passer que la stimulation de certains processus de période déterminée. Vraisemblablement, ils transfèrent ensuite à $\phi$ cette différence en communiquant au mouvement des neurones des périodes dont les différences sont de quelque façon analogues (énergie spécifique). Ce sont \{de telles\} modifications qui, au travers de $\phi$, puis de $\Psi$, se transmettent vers $P$, et qui, en y aboutissant presque dépourvues de quantité, produisent des sensations de qualités conscientes. Cette propagation de la qualité (Qualitätsfortpflanzung) n'est pas durable, elle ne laisse derrière elle aucune trace, elle n'est pas reproductible. 


\section{8 - LA CONSCIENCE}

Ce n'est qu'avec de telles hypothèses, complexes et peu concrètes, que j'ai réussi jusqu'à présent à faire entrer les phénomènes de la conscience dans l'édifice de la psychologie quantitative.

Nous ne tenterons naturellement pas d'expliquer pourquoi les processus d'excitation dans les neurones de perception $(\mathrm{N} \omega)$ entraînent la conscience. II ne s'agit que de faire correspondre aux propriétés de la conscience qui nous sont connues divers processus qui se déroulent parallèlement dans les neurones de perception $(\mathrm{N} \omega)$. Cela ne marche pas si mal que ça dans le détail. Un mot concernant les rapports entre cette théorie de la conscience et d'autres. Selon une théorie mécaniste moderne, la conscience est un simple accessoire qui vient s'ajouter aux processus pycho-physiologiques et dont l'élimination ne changerait rien au déroulement psychique. Selon une autre doctrine, la conscience constitue l'aspect subjectif de tout événement psychique, est donc inséparable du processus psychophysiologique. La doctrine développée entre les deux théories se situe ici. D'après elle, la conscience constitue l'aspect subjectif d'une partie des processus psychiques dans le système neuronique, c'est-à-dire des processus de perception (processus $\omega$ ), et l'élimination de la conscience ne laisse pas inchangé l'événement psychique mais implique l'élimination de la contribution qu'apporte le système $\mathrm{P}(\omega)$.

Si l'on représente la conscience par des neurones de perception $(\mathrm{N} \omega)$, cela a plusieurs conséquences, II faut que ces neurones aient une décharge, si petite soitelle, et il faut qu'il y ait un moyen de combler les neurones de perception par les quantités minimes $(\mathrm{Q} \eta)$ requises. La décharge, comme toujours, prend la voie de la motilité et à ce propos il faut remarquer que dans la transposition motrice chaque caractère de qualité, chaque particularité de la période se perd manifestement. Le comblement par la quantité des neurones de perception ne peut, il est vrai, se produire qu'à partir de $\Psi$, puisque nous ne voudrions attribuer à ce troisième système aucun nouage (Verknüpfung) direct avec $\phi$. On ne peut pas indiquer ce qu'était la valeur biologique originaire des neurones de perception.

Cependant nous n'avons donné jusqu'ici qu'une description incomplète du contenu de la conscience. II montre, outre les séries des qualités sensorielles, une autre série qui en est très différente et dont il nous faut maintenant donner une interprétation : celle des sensations de plaisir et de déplaisir. Puisqu'une tendance de la vie psychique, celle à éviter du déplaisir, est certaine, nous sommes tentés de l'identifier avec la tendance primaire à l'inertie. En ce cas, le déplaisir coïnciderait avec une élévation du niveau de la quantité $(\mathrm{Q} \eta)$ ou une augmentation de la pression exercée par les quantités; la perception serait la sensation liée à l'augmentation de la quantité $(Q \eta)$ en $\Psi$. Le plaisir serait la sensation de décharge. Étant donné que le système $P$ doit être comblé à partir de $\Psi$, il s'ensuivrait l'hypothèse que, lors d'une élévation de niveau en $\Psi$, l'investissement en $P$ augmente; lors d'une baisse de niveau, en revanche, il diminue. Plaisir et déplaisir seraient les sensations de l'investissement et du niveau propres à $P$.

$P$ et $\Psi$ représenteraient en quelque sorte des vases communicants. C'est aussi de cette manière que les processus quantitatifs en parviendraient à la conscience, encore une fois en tant que qualités.

Avec la sensation de plaisir et de déplaisir s'évanouit l'aptitude à percevoir des qualités sensorielles qui se situent pour ainsi dire dans la zone d'indifférence entre 
plaisir et déplaisir. Ceci serait à traduire comme suit: les neurones de perception ( $\mathrm{N}$ $\omega)$, lors d'un certain investissement, atteignent un point optimum dans la réception de la période du mouvement des neurones, ils produisent du déplaisir lors d'un investissement plus fort, du plaisir lors d'un investissement plus faible, jusqu'à ce que la capacité de réception disparaisse avec le manque d'investissement. Pour compléter ces données, il faudrait construire la forme de mouvement correspondante.

\section{9 - LE FONCTIONNEMENT DE L'APPAREIL}

On peut maintenant construire la représentation suivante du travail de l'appareil formé par $\phi \Psi \omega$. De l'extérieur, les grandeurs d'excitation assaillent les extrémités du système $\phi$, se heurtent d'abord aux appareils de terminaison nerveuse et sont fractionnés par ceux-ci en quotients qui sont probablement d'un ordre supérieur à celui des stimulations intercellulaires (peut-être quand même du même ordre ?). II y a ici un premier seuil : en-dessous d'une certaine quantité, on n'obtient pas de quotient efficace, de sorte que l'efficacité des stimulations est pour ainsi dire limitée aux quantités moyennes. De plus, la nature des gaines des terminaisons nerveuses agit comme tamis de manière que ce ne soit pas n'importe quelle stimulation qui puisse agir sur chacune des terminaisons. Les stimulations qui atteignent effectivement les neurones y ont une quantité et un caractère qualitatif, elles forment dans le monde extérieur une série de qualité égale et de quantité croissante, du seuil jusqu'à la limite de la douleur.

Tandis que dans le monde extérieur les processus représentent un continu dans deux directions, la quantité et la période (qualité), les stimulations qui leur correspondent sont, quant à la quantité, premièrement réduites, deuxièmement limitées par une coupure, et quant à la qualité, elles sont discontinues de sorte que certaines périodes n'agissent nullement comme stimulations.

Le caractère de qualité des stimulations se propage maintenant sans entrave par $\phi$ à travers $\Psi$ jusqu'à $\omega$ où il produit de la sensation. Il est représenté par une période particulière de mouvement neuronique qui n'est certainement pas la même que celle de la stimulation, mais qui a toutefois avec elle une certaine relation suivant une formule de réduction qui nous est inconnue. Cette période ne se maintient pas longtemps et s'évanouit vers le côté moteur : du fait qu'elle puisse passer, elle ne laisse pas non plus de mémoire.

La quantité de la stimulation $\phi$ excite la tendance à la décharge du système nerveux en se transposant en une excitation motrice proportionnelle. L'appareil de la motilité est appendu directement à $\phi$ et les quantités ainsi traduites créent un effet qui leur est quantitativement largement supérieur lorsqu'elles pénètrent dans les muscles, les glandes, etc..., donc elles y agissent par déliaison (Entbindung), tandis qu'entre les neurones il n'y a que du transfert (Ubertragung).

Les neurones $\Psi$ se terminent en outre dans les neurones $\phi$, une partie de la quantité $(Q \eta)$ leur est transférée, mais seulement une partie, peut-être bien un quotient qui correspond à une grandeur de stimulation intercellulaire. D'où la question: la quantité transférée sur $\Psi$ ne croit-elle pas proportionnellement à la quantité s'écoulant en $\phi$ de sorte qu'une stimulation plus grande exercerait un effet psychique plus fort? Ici, il semble y avoir un dispositif particulier qui de nouveau écarte de la quantité $(Q)$ de $\Psi$. La conduction $\phi$ sensible est, en effet, construite d'une façon singulière, elle se ramifie continuellement et présente des voies plus ou 
moins grosses ou minces qui aboutissent à de nombreuses terminaisons, avec probablement la signification suivante: une stimulation plus forte emprunte d'autres chemins qu'une stimulation plus faible. $(Q \eta) 1$, par exemple, n'empruntera que la voie I et transférera un quotient en $\Psi$ à la terminaison $\alpha .(Q \eta) 2$ ne transférera pas un quotient double en $\alpha$, mais pourra aussi emprunter le chemin II qui est plus étroit et

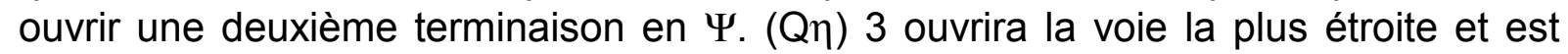
aussi transférée par $\gamma$. C'est ainsi que chaque voie $\phi$ est délestée, et qu'une plus grande quantité en $\phi$ s'exprimera en investissant en $\Psi$ plusieurs neurones au lieu d'un seul. Chaque investissement des neurones $\Psi$ peut en cela être approximativement identique. Si $Q \eta$ en $\phi$ donne un investissement en $\Psi,(Q \eta) 3$ s'exprime par un investissement en $\Psi 1+\Psi 2+\Psi 3$. La quantité en $\phi$ s'exprime donc par la complication en $\Psi$. En cela, la quantité $(Q)$ est écartée de $\Psi$, au moins jusqu'à certaines limites. Ceci rappelle beaucoup les rapports de la loi de Fechner qui se laisserait ainsi localiser.
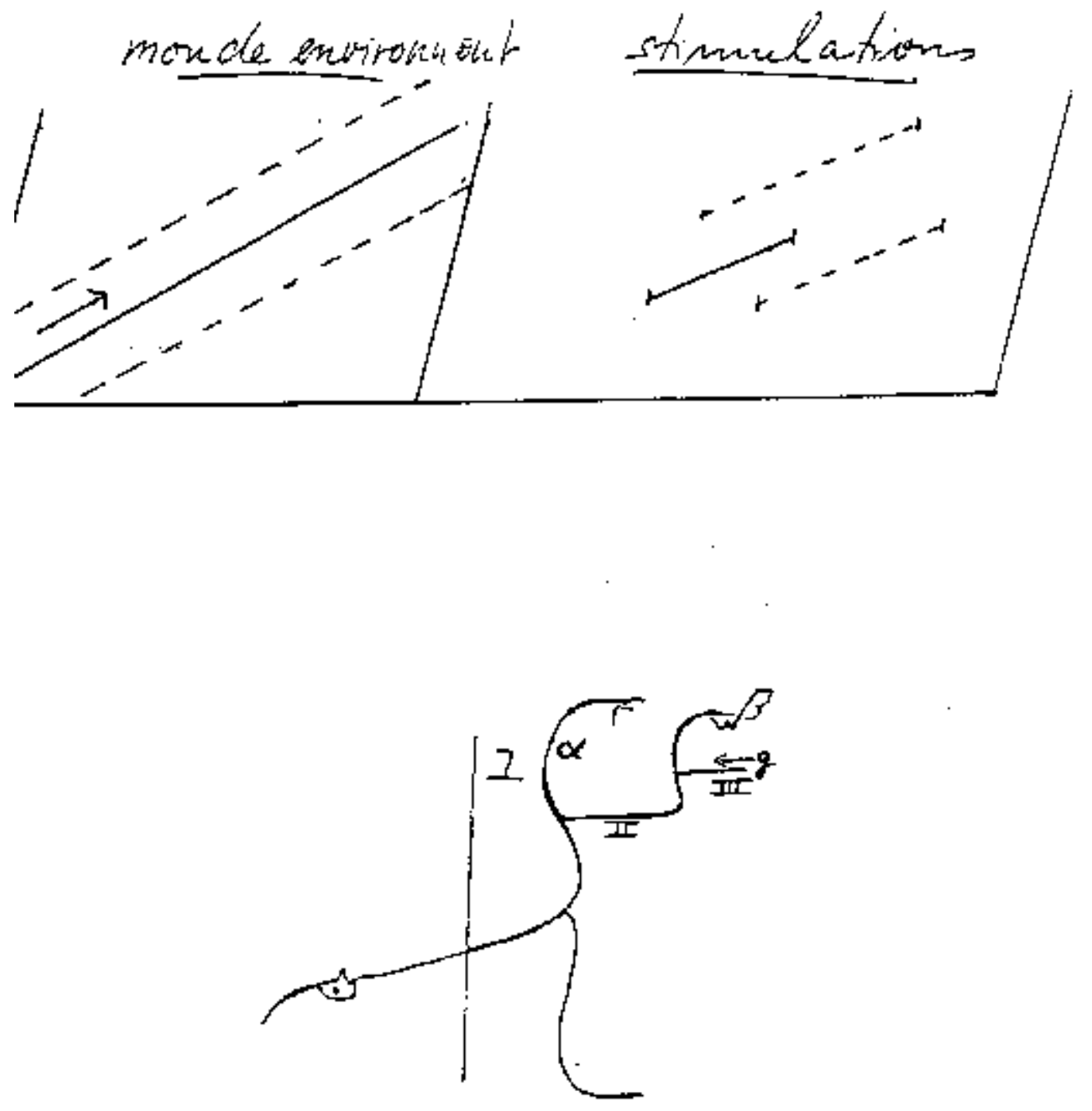
De cette manière, $\Psi$ est investi à partir de $\phi$ en quantités (Q) qui, normalement, sont petites. La quantité de l'excitation en $\phi$ s'exprime en $\Psi$ par la complication, la qualité par la topique; en effet, selon les relations anatomiques, chaque organe sensoriel n'est mis en rapport par $\phi$ qu'avec des neurones $\Psi$ déterminés. $\Psi$ reçoit pourtant encore de l'investissement à partir de l'intérieur du corps, et il est possible de décomposer les neurones $\Psi$ en deux groupes, les neurones du pallium qui sont investis à partir de $\phi$, et les neurones du noyau qui sont investis à partir de conductions endogènes.

\section{0 - LES CONDUCTIONS $\Psi$}

Le noyau de $\Psi$ est en liaison avec les voies sur lesquelles des quantités d'excitations endogènes s'établissent. Sans exclure de liaisons de ces voies avec $\Phi$, il nous faut quand même retenir l'hypothèse de départ qu'un chemin direct mène de l'intérieur du corps à des neurones $\Psi$. Mais alors $\Psi$ est exposé de ce côté sans protection aux quantités $(Q)$, et là se trouve le ressort pulsionnel (Triebfader) du mécanisme psychique.

Ce que nous savons des stimulations endogènes peut se formuler par l'hypothèse qu'elles sont de nature intercellulaire, s'établissant de façon continue et ne se transforment que périodiquement en stimulations psychiques. L'idée d'une accumulation est irréfutable et l'intermittence de l'effet psychique autorise seulement à penser qu'elles se heurtent sur leur chemin conducteur vers $\Psi$ à des résistances qui ne sont surmontées que lors de l'accroissement de la quantité. Il s'agit donc de conductions à articulations multiples, avec interposition de plusieurs barrières de contact jusqu'au noyau $\Psi$. Mais à partir d'une certaine quantité $(Q)$, elles agissent en permanence comme stimulation et chaque augmentation de la quantité ( $Q$ ) est perçue comme augmentation de la stimulation $\Psi$. II y a donc un état dans lequel la conduction est devenue perméable. L'expérience apprend de plus qu'après décharge de la stimulation $\Psi$ la conduction reprend à nouveau sa résistance.

On appelle un tel processus: sommation. Les conductions $\Psi$ se comblent par sommation jusqu'à ce qu'elles deviennent perméables. II est patent que c'est la petitesse de chaque stimulation qui permet la sommation. II s'avère qu'il y a aussi de la sommation pour les conductions $\Phi$, par exemple pour la conduction de la douleur, là aussi seulement pour de petites quantités. Le moindre rôle de la sommation du côté de $\Phi$ plaide pour ceci: qu'il s'y agit en fait de plus grandes quantités. De très petites quantités semblent être tenues à l'écart par l'effet de seuil des appareils de terminaison nerveuse, tandis que du côté de $\Psi$ de tels appareils manquent, et n'y agissent que de petites quantités.

II est à noter que les neurones de conduction $\Psi$ peuvent se maintenir entre les caractéristiques de la perméabilité et de l'imperméabilité, et qu'ils reprennent à nouveau la presque totalité de leur résistance malgré le passage de la quantité $(Q \eta)$. Ceci contredit entièrement la propriété supposée des neurones $\Psi$ d'être frayés en permanence par l'écoulement de la quantité $(Q \eta)$. Comment expliquer cette contradiction ? Par l'hypothèse que le rétablissement de la résistance lors de la cessation de l'écoulement est une propriété générale des barrières de contact. Cela s'accorde sans difficulté avec le fait que les neurones sont influencés dans le sens du frayage. On a seulement besoin de supposer que le frayage qui reste après l'écoulement de quantité ne consiste pas dans la levée de chaque résistance, mais 
dans la diminution de celle-ci jusqu'à un minimum restant nécessaire Pendant l'écoulement de quantité (Q), la résistance est levée, ensuite elle ne se rétablit qu'à une hauteur différente selon la quantité (Q) écoulée, ce qui fait que la fois suivante une moindre quantité (Q) peut passer, etc... Lors du frayage le plus complet, une certaine résistance de toutes les barrières de contact demeure, qui exige donc aussi un accroissement de quantité $(Q)$ jusqu'à un certain seuil pour que celle-ci passe. Cette résistance serait une constante. Ainsi le fait que les quantités endogènes $(Q \eta)$ opèrent par sommation, ne signifie rien d'autre que ceci : ces quantités se composent de grandeurs d'excitation très petites se situant en dessous de la constante ; la conduction endogène est ainsi néanmoins complètement frayée.

Il en résulte pourtant que les barrières de contact $\Psi$ s'élèvent en général plus haut que les barrières de conduction, de sorte que dans les neurones du noyau une nouvelle accumulation de quantité $(Q \eta)$ puisse avoir lieu. Aucune autre limite ne lui est posée à partir de l'égalisation de la conduction. $\Psi$ est ici livré à la quantité $(Q)$, et à l'intérieur du système s'établit ainsi l'impulsion qui entretient toute activité psychique. Nous connaissons cette puissance en tant que volonté: le rejeton des pulsions.

\section{1- L'EVENEMENT DE SATISFACTION (DAS BEFRIEDIGUNGSERLEBNIS)}

Le comblement des neurones du noyau en $\Psi$ a pour conséquence un effort vers une décharge, une poussée qui s'évacue vers la voie motrice. Selon l'expérience, c'est la voie du changement interne qui est d'abord empruntée (expression du mouvement d'âme, cri, innervation des vaisseaux). Toute décharge de cet ordre n'aura pourtant, comme notre introduction l'expose, aucun résultat qui soulage puisque l'absorption de stimulation endogène persiste quand même et qu'elle rétablit la tension $\Psi$. Une levée de stimulation n'y est possible que par une intervention qui, à

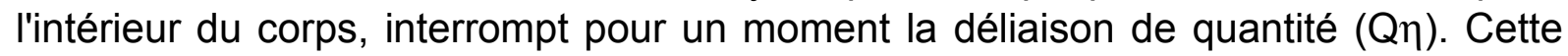
intervention exige un changement dans le monde extérieur (apport de nourriture, proximité de l'objet sexuel) qui ne peut se produire, en tant qu'action spécifique, que par des chemins déterminés. L'organisme humain est d'abord incapable de mettre en œuvre l'action spécifique. Elle se produit par aide étrangère, quand, par la décharge par voie de changement interne, l'attention d'une personne d'expérience est attirée sur l'état de l'enfant. Cette voie de décharge prend ainsi la fonction secondaire très importante de la communication et la détresse (1) initiale de l'être humain est la source originaire de tous les motifs moraux.

Quand l'individu secourable a accompli le travail de l'action spécifique dans le monde extérieur pour l'individu en détresse, celui-ci est capable, par dispositifs réflexes, de réaliser sans peine le travail nécessaire à la levée de stimulation endogène à l'intérieur de son corps. Le tout représente alors un événement de satisfaction qui a les conséquences les plus marquantes pour le développement fonctionnel de l'individu. II se passe trois choses dans le système $\Psi$. 1) Une décharge durable s'accomplit, et il est ainsi mis fin à la poussée qui a créé du déplaisir en $\mathrm{P}$; 2) dans le pallium s'établit l'investissement d'un neurone (ou de plusieurs neurone) qui correspondent à la perception d'un objet; 3) les informations sur la décharge provoquées par le mouvement-réflexe déclenché après l'action spécifique arrivent à d'autres lieux du pallium. Entre ces investissements et les neurones du noyau se forme un frayage. 
Les informations sur la décharge-réflexe se produisent parce que chaque mouvement, par ses conséquences annexes, est l'occasion de nouvelles excitations sensibles (de la peau et des muscles). Le frayage se forme pourtant d'une manière qui permet de mieux saisir le développement de $\Psi$. Jusqu'à présent nous avons appris à connaître comment les neurones sont influencés par les neurones $\Psi$ et les conductions endogènes; chaque neurone était pourtant séparé des autres par des barrières de contact à fortes résistances. Maintenant, il existe une loi fondamentale de l'association par simultanéité qui agit lors de l'activité $\Psi$ pure, lors de la remémoration reproductrice, et qui est le fondement de toutes les liaisons entre les neurones $\Psi$. Nous constatons que la conscience, donc l'investissement quantitatif, passe d'un neurone $\Psi \alpha$ à un deuxième neurone $\Psi \beta$, lorsque $\alpha$ et $\beta$ ont été une fois investis simultanément à partir de $\Phi$ (ou d'où que ce soit). C'est donc par un investissement simultané $\alpha \beta$ qu'une barrière de contact a été frayée. II en découle, dans les expressions de notre théorie, qu'une quantité venant d'un neurone passe plus facilement dans un neurone investi que dans un neurone non investi.

L'investissement du deuxième neurone agit donc comme renforcement de l'investissement du premier. L'investissement se montre ici à nouveau comme équivalent au frayage pour l'écoulement de la quantité.

Nous apprenons donc ici à connaître un deuxième facteur important pour !a direction de l'écoulement de quantité. Une quantité dans le neurone $\alpha$ ne prendra pas seulement la direction de la barrière la mieux frayée, mais aussi de celle investie par le côté opposé. Les deux facteurs peuvent se soutenir l'un l'autre, ou éventuellement agir l'un contre l'autre.

Par expérience de satisfaction, il s'établit donc un frayage entre deux images de souvenir et les neurones du noyau qui sont investis en état d'urgence. Avec la décharge de satisfaction, la quantité $(Q \eta)$ est bien sûr entraînée hors des images de souvenir. Avec le retour de l'état d'urgence ou de vœu, l'investissement passe maintenant aussi aux deux souvenirs et les anime. C'est probablement d'abord l'image de souvenir d'objet qui est concernée par la reviviscence du vœu.

Je ne doute pas que cette reviviscence du vœu donne d'abord la même chose que la perception, c'est-à-dire une hallucination. Si l'action réflexe est ensuite amorcée, la désillusion ne fait pas défaut.

\section{2 - L'EVENEMENT DE DOULEUR}

$\Psi$ est normalement exposé à la quantité $(Q \eta)$ à partir des conductions endogènes; mais il l'est de façon anormale -quand bien même pas encore pathologique- pour le cas où des quantités $(Q)$ pas trop grandes percent les dispositifs-écran en $\Phi$, donc dans le cas de la douleur. La douleur crée en $\Psi: 1)$ une grande augmentation de niveau, qui est ressentie par $\mathrm{P}$ comme déplaisir; 2) une pente à l'évacuation qui peut être modifiée selon certaines directions; 3 ) un frayage entre cette dernière et une image de souvenir de l'objet provoquant la douleur. II est par ailleurs certain que la douleur a une qualité particulière qui se fait valoir à côté du déplaisir.

Si l'image de souvenir de l'objet (hostile) est d'une quelconque façon investie de nouveau, par exemple par des perceptions nouvelles, alors un état s'établit qui n'est pas de la douleur, mais lui ressemble. II contient du déplaisir et la pente à l'évacuation qui correspond à l'événement de douleur. Puisque déplaisir signifie augmentation de niveau, la question de l'origine de cette quantité $(\mathrm{Q} \eta)$ se pose. 
Dans l'événement de douleur proprement dit, c'était la quantité extérieure (Q), faisant irruption, qui augmentait le niveau. Dans sa reproduction -dans l'affect- n'a été ajoutée que la quantité qui investit le souvenir et il est clair que celle-ci, de même nature que la perception, ne peut pas avoir comme conséquence une augmentation générale de quantité $(Q \eta)$.

II ne reste donc que l'hypothèse selon laquelle, par l'investissement de souvenir, du déplaisir est délié à l'intérieur du corps, nouvellement extrait. On peut se représenter le mécanisme de cette déliaison comme suit: de même qu'il y a des neurones moteurs qui, pour un certain comblement, conduisent des quantité $(\mathrm{Q} \eta)$ dans les muscles et les évacuent ainsi, de même il faut qu'il y ait aussi des neurones "sécréteurs" qui, quand ils sont excités, permettent que s'établissent à l'intérieur du corps ce qui agit comme stimulation sur les conductions endogènes vers $\Psi$. Ils influencent donc la production de quantités endogènes $(\mathrm{Q \eta})$, mais loin de les évacuer, ils les ramènent en passant par des voies de détour.

Nous nommerons ces neurones des "neurones-clefs". II est patent qu'ils ne sont excités que lors d'un certain niveau en $\Psi$. Par l'événement de douleur, l'image de souvenir de l'objet hostile a obtenu un frayage exemplaire vers ces neurones-clefs grâce auxquels se délie maintenant du déplaisir dans l'affect.

Le comportement de la déliaison sexuelle nous fournit un étayage pour cette hypothèse étrange mais indispensable. La présomption que les stimulations endogènes consisteraient ici et là en des produits chimiques dont le nombre pourrait être considérable, s'impose en même temps. Puisque la déliaison du déplaisir pour un investissement tout à fait minime du souvenir hostile peut être une déliaison extraordinaire, on peut en conclure que la douleur laisse derrière elle des frayages particulièrement abondants. Le frayage -on le devine- dépend de bout en bout de la quantité obtenue, de sorte que l'effet de frayage de $3 Q \eta$ pourrait largement surpasser celui de $3 \times \mathrm{Q \eta}$.

\section{3 - LES AFFECTS ET ETATS DE VCEU}

Les restes des deux sortes d'événements ici traités sont les affects et les états de vœux qui ont en commun de comporter une élévation de la tension de la quantité en $\Psi$ produite, dans le cas de l'affect, par déliaison brusque, dans le cas du vœu par sommation. Les deux états sont de la plus grande importance pour l'écoulement en $\Psi$ puisqu'ils laissent des motifs quasi contraignants pour cet écoulement. De l'état de vœu découle directement une attraction vers l'objet de vœu, c'est-à-dire vers son image de souvenir; de l'événement de douleur résulte une répulsion, une aversion à maintenir investie l'image de souvenir hostile. II s'agit ici de l'attraction primaire du vœu et de la défense primaire.

On peut facilement s'expliquer l'attraction du vœu par l'hypothèse selon laquelle, dans l'état de vœu, l'investissement de l'image de souvenir- aimable dépasse largement en quantité $(\mathrm{Q} \eta)$ l'investissement réalisé dans une simple perception; de sorte qu'un frayage particulièrement bon conduit du noyau $\Psi$ vers le neurone correspondant au pallium.

II est plus difficile d'expliquer la défense primaire ou refoulement, à savoir le fait qu'une image de souvenir hostile est toujours le plus vite possible laissée en plan par l'investissement. Néanmoins l'explication pourrait résider en ceci qu'il a été mis fin aux événements primaires de douleur par défense réflexe. Le surgissement d'un 
autre objet à la place de l'objet hostile a été le signal que l'événement de douleur était fini et le système $\Psi$, instruit par le biologique, essaie de reproduire en $\Psi$ l'état qui marquait l'arrêt de la douleur. Par l'expression "instruit par le biologique", nous avons introduit une nouvelle base d'explication qui doit avoir sa validité propre bien qu'elle n'exclut pas un retour aux principes mécaniques (facteurs quantitatifs), mais qu'elle l'exige. Dans le cas présent, ce pourrait bien être l'augmentation de quantité $(\mathrm{Q} \eta)$ se produisant à chaque investissement de souvenirs hostiles qui pousse à une activité d'évacuation augmentée, donc aussi à l'écoulement de souvenirs.

\section{4 - INTRODUCTION DU "MOI"}

En fait, avec l'hypothèse de l' "attraction de vœu" et la pente vers le refoulement, nous avons déjà abordé un état de $\Psi$ qui n'a pas encore été discuté, car ces deux processus indiquent qu'une organisation s'est formée en $\Psi$ dont la présence perturbe des écoulements qui, la première fois, se sont accomplis de manière déterminée. Cette organisation s'appelle le "Moi" et peut facilement être figurée en considérant que la réception régulièrement répétée de quantités endogènes dans des neurones déterminés (du noyau) et l'effet de frayage qui en découle donneront un groupe de neurones investis de façon constante, qui correspond donc au magasin à provisions exigé par la fonction secondaire. Le Moi est donc à définir comme la totalité des investissements $\Psi$ à un moment donné, parmi lesquels on doit distinguer un élément permanent et un élément transitoire. Comme on le comprend facilement, Le frayage entre neurones $\Psi$ est une caractéristique du Moi, en tant que possibilité d'assigner au Moi modifié son extension, à tout moment.

L'effort du Moi doit nécessairement être de se débarrasser de ses investissements par la voie de la satisfaction. II ne peut le faire qu'en influençant la répétition des événements de douleur et des affects par la voie suivante, généralement désignée comme celle de l'inhibition

Une quantité $(\mathrm{Q \eta})$ qui, à partir de n'importe où, fait irruption dans un neurone, se propagera vers la barrière de contact du plus grand frayage et provoquera vers lui un courant. Plus précisément, le flux de quantité $\left(Q_{\eta}\right)$ se distribuera vers chacune des barrières de contact en proportion inverse de la résistance, et là où une barrière de contact est atteinte par un quotient qui subit sa résistance, pratiquement rien ne passera. Ce rapport peut facilement varier dans le neurone pour chaque quantité $(\mathrm{Q} \eta)$, car les quotients peuvent se former qui dépassent largement le seuil d'autres barrières de contact. L'écoulement est donc dépendant des quantités $(Q \eta)$ et du rapport des frayages. Mais nous avons appris à connaître le troisième et très important facteur. Quand un neurone contigu est investi simultanément, alors ceci agit comme un frayage passager des barrières de contact situées entre les deux neurones et modifie l'écoulement qui, autrement, se serait dirigé vers la seule barrière de contact frayée. Un investissement latéral est donc une inhibition pour l'écoulement de la quantité $(\mathrm{Q \eta})$. 


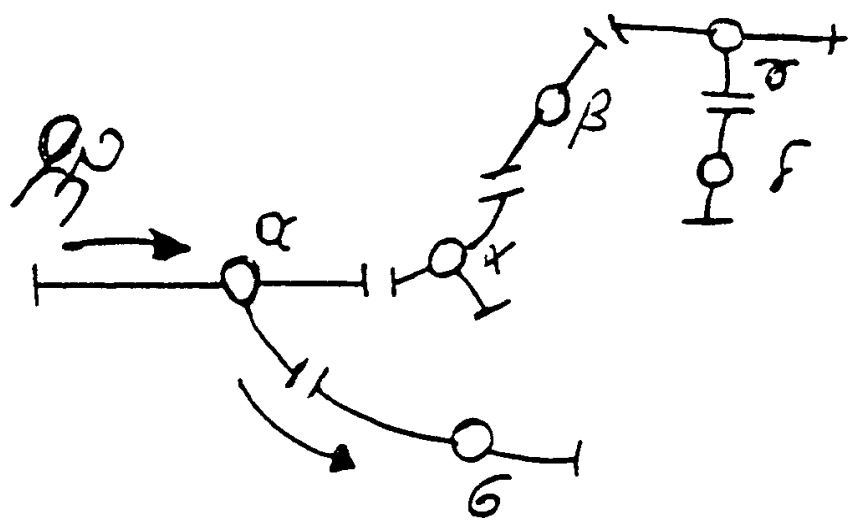

Représentons-nous le Moi comme un réseau de neurones investis et bien frayés les uns par rapport aux autres; soit une quantité $(Q \eta)$ qui pénètre en $\alpha$ à partir de l'extérieur $(\Phi)$ et qui, non influencé, serait allé vers $\beta$; si elle est influencée par l'investissement latéral $\alpha$ en a, alors elle ne cède qu'un quotient vers $\beta$ ou, éventuellement, elle n'atteint pas du tout $b$. Donc si un Moi existe, il faut qu'il inhibe des processus psychiques primaires. Or une telle inhibition est un avantage décisif pour $\Psi$. Supposons que $\alpha$ soit un souvenir hostile, $\beta$ un neurone-clef vers le déplaisir; alors, à l'éveil de $\alpha$, du déplaisir sera primairement délié, déplaisir qui sera peut-être sans but, qui l'est de toute façon si la déliaison s'effectue selon la totalité de son montant. S'il y a effet d'inhibition de $\alpha$, alors la déliaison de déplaisir sera très faible, et seront épargnés au système neuronique le développement et l'évacuation de quantité sans qu'il en résulte d'autre dommage. On peut maintenant facilement se représenter qu'avec l'aide du mécanisme qui attire l'attention du Moi sur le nouvel investissement intervenant alors de l'image hostile de souvenir, le Moi peut parvenir, par un large investissement latéral renforcé au besoin, à inhiber l'écoulement à partir de l'image de souvenir vers la déliaison de déplaisir. Et même, à supposer que la déliaison de déplaisir initiale $(\mathrm{Q} \eta)$ soit absorbée par le Moi lui-même, nous trouvons précisément en celle-ci la source de la dépense qu'exige l'investissement latéral inhibant venant du Moi.

La défense primaire est donc d'autant plus forte qu'est plus fort le déplaisir.

\section{5 - PROCESSUS PRIMAIRE ET PROCESSUS SECONDAIRE EN $\Psi$}

II résulte des développements précédents que -du point de vue de ses tendancesnous pouvons traiter le Moi en comme le système nerveux dans son ensemble. II tombe en état de détresse et subit des dommages lors de processus non influencés en $\Psi$, et ceci dans deux cas. A savoir-, premièrement quand, dans l'état de vœu, il investit à nouveau le souvenir d'objet, puis laisse se produire la décharge alors que la satisfaction va nécessairement faire défaut parce que l'objet n'est pas réel, mais qu'il n'est présent que dans la représentation de fantasme. $\Psi$ est d'abord hors d'état de faire cette différenciation parce qu'il ne peut travailler qu'en s'appuyant sur une série d'états analogues entre ses neurones. II a donc besoin d'un critère provenant d'ailleurs pour différencier perception et représentation (Vorstellung).

D'autre part, $\Psi$ a besoin d'un signe pour être attentif au ré-investissement de l'image de souvenir hostile et pour prévenir par investissement latéral la déliaison de 
déplaisir qui s'en suit. Lorsque $\Psi$ peut réaliser cette inhibition à temps, la déliaison de déplaisir, et donc la défense, tombent à un faible niveau; dans l'autre cas, il y a un énorme déplaisir et une défense primaire excessive.

L'investissement de vœu, comme la déliaison de déplaisir lors du nouvel investissement du souvenir concerné, peuvent être biologiquement nuisibles. L'investissement de vœu l'est chaque fois qu'il dépasse une certaine mesure et incite par là à la décharge; la déliaison de déplaisir l'est au moins chaque fois que l'investissement de l'image de souvenir hostile ne provient pas du monde extérieur mais de $\Psi$ lui-même (par association). II s'agit donc ici aussi d'un signe pour distinguer la perception du souvenir (représentation) (Vorstellung).

Vraisemblablement ce sont alors les neurones de perception qui fournissent ce signe, le signe de réalité. Pour chaque perception externe s'établit en $P$ une excitation porteuse de qualité, qui pourtant est d'abord sans signification pour $\Psi$. II faut encore ajouter que l'excitation par la perception conduit à une décharge propre à la perception et que, à partir de celle-ci comme à partir de toute décharge, une information aboutit à $\Psi$. L'information de décharge venant de $\mathrm{P}(\omega)$ est alors pour $\Psi$ le signe de qualité ou de réalité.

Si l'objet de vœu est investi abondamment, au point d'être animé hallucinatoirement, il s'ensuit également le même signe d'évacuation ou de réalité que pour la perception externe. Dans ce cas, le critère fait défaut. Mais si l'investissement de vœu a lieu sous inhibition, comme c'est possible pour un Moi investi, alors est concevable un cas quantitatif où, l'investissement de vœu n'étant pas assez intense, il ne donne aucun signe de réalité alors que la perception externe en donnerait un. Pour ce cas, le critère garde donc sa valeur. En effet, la différence tient à ce que le signe de qualité a lieu à partir de l'extérieur pour chaque intensité d'investissement, mais n'a lieu à partir de $\Psi$ que pour de grandes intensités. C'est donc l'inhibition pour le Moi qui rend possible un critère pour la différenciation entre perception et souvenir. L'expérience biologique apprendra alors à ne pas amorcer la décharge avant que le signe de réalité soit arrivé et, à cette fin, apprendra à ne pas pousser au-delà d'une certaine mesure l'investissement des souvenirs sur lesquels porte le vœu.

D'autre part, l'excitation des neurones de perception peut aussi servir à protéger le système $\Psi$ dans le second cas, l'attention de $\Psi$ étant alors attirée sur le fait qu'il y a ou qu'il n'y a pas une perception. A cette fin, il faut supposer que les neurones de perception $(\mathrm{N} \omega)$ sont à l'origine en liaison anatomique avec les voies de conduction des différents organes sensoriels et dirigent à nouveau leur décharge sur des appareils moteurs qui appartiennent aux mêmes organes sensoriels. Alors cette dernière information (celle de l'attention réflexe) deviendra, pour $\Psi$, un signal d'envoyer par la voie biologique des quantités d'investissement dans les mêmes directions.

Donc: lors d'une inhibition par le Moi investi, les signes de décharge issus de $\omega$ deviennent de façon tout à fait générale des signes de réalité, que $\Psi$ apprend biologiquement à utiliser. Si le Moi se trouve, lors du surgissement d'un tel signe de réalité, en état de tension de vœu, alors il laissera la décharge s'ensuivre, orientée vers l'action spécifique; si une augmentation de déplaisir coincide avec le signe de réalité, alors $\Psi$, par un grand investissement latéral approprié à l'endroit indiqué, mettra en place une défense d'intensité normale. Hors de ces deux cas, 
l'investissement pourra avoir lieu sans entrave en suivant les rapports de frayage. Nous désignons l'investissement de vœu jusqu'à l'hallucination, le plein développement du déplaisir qui entraîne avec lui la pleine dépense de défense, comme processus psychiques primaires; par contre, ces processus qui ne sont rendus possibles que par le bon investissement du Moi et représentent une atténuation des précédents, nous les désignons comme processus psychiques secondaires. La condition de ces derniers est, comme on voit, une juste utilisation des signes de réalité qui n'est possible que lors de l'inhibition par le Moi.

\section{6 - LE PENSER QUI RECONNAIT ET LE PENSER QUI REPRODUIT}

Après avoir introduit l'hypothèse, lors du processus de vœu, que l'inhibition venant du Moi amène un investissement tempéré de l'objet de vœu qui donne lieu à, sa reconnaissance comme non réelle, nous pouvons poursuivre l'analyse de ce processus. Plusieurs cas peuvent se produire. Premièrement: l'investissement de vœu de l'image de souvenir et la perception de celle-ci sont simultanément présents; alors les deux investissements coïncident, ce qui n'est pas utilisable sur le plan biologique, mais en outre, se produit à partir de $\mathrm{P}$ le signe du réel après lequel, conformément à l'expérience, la décharge réussit. Ce cas est facilement réglé. Deuxièmement: l'investissement de vœu est présent, et à côté il y a une perception qui coïncide avec celui-ci non pas entièrement mais seulement partiellement. En effet, il est temps de se souvenir que les investissements de perception ne sont jamais des investissements de neurones isolés, mais toujours de complexes de neurones. Nous avons jusque là négligé ce trait; il est maintenant temps d'en tenir compte. Supposons que l'investissement de vœu concerne de façon tout à fait générale neurone $\mathbf{a}+$ neurone $\mathbf{b}$ tandis que les investissements de perception concernent neurone $\mathbf{a}+$ neurone $\mathbf{c}$. Comme ce sera là le cas le plus fréquent, plus fréquent que celui de l'identité, il exige un examen plus précis. L'expérience biologique nous apprendra ici aussi qu'il est incertain d'ouvrir la voie à la décharge quand les signes de réalité confirment non pas le complexe tout entier, mais seulement une partie de celui-ci. Mais une voie est maintenant trouvée pour parfaire la ressemblance avec l'identité. Le complexe $\mathrm{P}$, par comparaison avec d'autres complexes $P$, se décomposera justement en un élément neurone a qui, le plus souvent, reste identique à lui-même, et en un second élément neurone b qui, la plupart du temps, varie. Par la suite la langue instituera le terme jugement (Urteil: partition originaire) pour désigner cette décomposition et trouvera la ressemblance qui se pose en effet entre le noyau du Moi et l'élément de perception constant, entre les investissements changeants dans le pallium et l'élément inconstant; elle nommera le neurone a la chose et le neurone $\mathbf{b}$ son activité ou sa propriété, bref son prédicat.

Le juger est donc un processus $\Psi$ qui n'est rendu possible que grâce à l'inhibition venant du Moi, et qui est provoqué par la dissemblance entre l'investissement de vœu d'un souvenir et un investissement de perception qui lui .ressemble. On peut prendre pour point de départ que la coïncidence entre les deux investissements devient le signal biologique selon lequel il faut mettre un terme à l'acte de penser et laisser s'ouvrir la voie à la décharge. La séparation donne l'impulsion au travail de penser qui prendra fin à nouveau avec la coïncidence.

On peut pousser plus loin l'analyse du processus: lorsque le neurone a coïncide, mais que c'est neurone $\mathbf{c}$ qui est perçu au lieu de neurone $\mathbf{b}$, alors le travail du Moi 
s'exerce en suivant les liaisons de ce neurone $c$ et fait surgir de nouveaux investissements au moyen de l'envoi d'un flux de quantité le long de ces liaisons, jusqu'à ce que soit trouvé un accès au neurone manquant $\mathbf{b}$. En règle générale, se produit une image de mouvement qui est intercalée entre neurone $\mathbf{c}$ et neurone $\mathbf{b}$, et, avec la reviviscence de cette image grâce à un mouvement effectivement accompli, la perception de neurone $\mathbf{b}$ et avec elle l'identité recherchée sont établies. Par exemple, supposons que l'image de souvenir en cause dans le vœu soit la vue complète de l'image du sein maternel et de son mamelon, mais que la première perception donnée soit une vue de profil du même objet, sans le mamelon. Dans la mémoire de l'enfant se trouve une expérience, faite fortuitement pendant la tétée, de telle façon qu'avec un mouvement déterminé de la tête, l'image totale se transforme en image de profil. L'image de profil qui est vue maintenant conduit au mouvement de la tête, un essai montre que sa contrepartie doit être accomplie et la perception de la vue complète est alors acquise.

Ceci ne montre que peu du jugement, mais c'est un exemple de la possibilité d'arriver par la reproduction d'investissements à une action qui déjà appartient au côté accidentel de l'action spécifique.

II ne fait aucun doute que c'est la quantité ( $Q \eta)$ provenant du Moi investi, qui donne support à ces migrations le long des neurones frayés, et que cette migration n'est pas maîtrisée par les frayages, mais par un but. Quel est ce but et comment est-il atteint?

Le but est de retourner au neurone manquant $b$ et de déclencher la sensation d'identité, c'est donc le moment où seul le neurone $b$ est investi, et où l'investissement migrant débouche dans le neurone b. Ceci est obtenu grâce à un déplacement à titre d'essai de la quantité sur toutes les voies, et il est clair que pour cela est nécessaire tantôt une plus grande, tantôt une moindre dépense d'investissement latéral, selon qu'on peut se servir des frayages existants ou qu'il faut agir contre eux. Le combat entre les frayages stables et les investissements changeants caractérise le processus secondaire de la pensée par opposition à la série primaire d'associations.

Qu'est-ce qui régit cette migration ? Le fait que la représentation de vœu du souvenir est maintenue investie pendant que l'on suit la voie d'association à partir du neurone c. Nous savons que, grâce à un tel investissement du neurone b, toutes ses liaisons possibles deviennent elles-mêmes mieux frayées et plus accessibles.

Lors de cette migration, il peut arriver que la quantité $(\mathrm{Q} \eta)$ se heurte à un souvenir qui est en relation avec un événement de douleur et, par là, fournisse l'occasion d'une déliaison de déplaisir. Comme cela est un signe certain que neurone b n'est pas accessible sur cette voie, le courant se détourne aussitôt de l'investissement concerné. Mais Les voies de déplaisir conservent leur haute valeur pour diriger le courant de la reproduction.

\section{7 - LE REMÉMORER ET LE JUGER}

La pensée reproductrice a aussi une visse pratique et une fin biologique bien établie, à savoir ramener une quantité migrante $(Q \eta)$ de la perception excédante à l'investissement manquant de neurones. Alors l'identité et le droit à la décharge sont atteints, si le signe de réalité venant du neurone b apparaît encore. Mais le processus peut se rendre indépendant de cette dernière visée et ne tendre qu'à l'identité. On est alors en présence d'un pur acte de pensée mais qui, dans chaque 
cas, peut ultérieurement être rendu exploitable en pratique. De plus, le Moi investi s'y comporte d'une manière tout à fait identique.

Nous en venons à une troisième possibilité qui peut se produire dans l'état de vœu, à savoir que, en présence d'un investissement de vœu, une perception qui émerge ne coïncide pas du tout avec l'image du souvenir souhaitée. Alors se produit un intérêt à reconnaître cette image de perception pour éventuellement trouver tout de même à partir d'elle une voie vers $S$ + II est à supposer que, dans ce but, la perception se trouve surinvestie aussi à partir du Moi comme, dans le cas précédent, ne l'était que l'élément neurone c. Si la perception n'est pas absolument nouvelle, elle rappellera maintenant le souvenir d'une perception, réveillera celle-ci avec laquelle elle coïncide au moins en partie. Au niveau de cette image de souvenir se répète maintenant le processus de pensée de tout à l'heure. Mais dans une certaine mesure sans le but qu'offrait la représentation de vœu investie.

Dans la mesure où les investissements coïncident, ils n'incitent pas à un travail de pensée. Par contre, les parts qui se dissocient "éveillent l'intérêt" et peuvent inciter à deux sortes de travail de pensée. Soit le flux se dirige vers les souvenirs éveillés et met en marche un travail de souvenir sans but qui est donc mû par les différences et non par les ressemblances, soit il demeure dans les composantes nouvellement surgies et représente alors un travail de jugement également sans but.

Supposons que l'objet qui fournit la perception soit semblable au sujet, soit un semblable (Nebenmensch). L'intérêt théorique s'explique alors aussi par ceci qu'un tel objet est simultanément le premier objet de satisfaction, puis ultérieurement le premier objet hostile, tout comme l'unique puissance qui secourt. C'est auprès du semblable que l'homme apprend à reconnaître. Alors les complexes de perception qui partent de ce semblable seront en partie nouveaux et incomparables, ses traits, par exemple dans le domaine visuel; d'autres perceptions visuelles, par exemple celles de ses mouvements de main, coïncideront par contre dans le sujet avec le souvenir de ses propres impressions visuelles, tout à fait semblables, provenant de son propre corps, et avec lesquelles se trouvent en association les souvenirs de mouvements vécus par lui-même. D'autres perceptions encore de l'objet, par exemple lorsqu'il crie, éveilleront le souvenir de son propre crier, et, du même coup, des événements de douleur qui lui sont propres. Et ainsi le complexe du prochain se sépare en deux composantes dont l'un en impose par un montage constant, reste ensemble comme chose, tandis que l'autre peut être compris par un travail de remémoration, c'est-à-dire peut être ramené à une information venant du corps propre. Cette décomposition d'un complexe de perception c'est le reconnaître, elle contient un jugement et prend fin quand ce dernier but est atteint. Le jugement n'est, comme on le voit, pas une fonction primaire, mais présuppose l'investissement de la part disparate du Moi; d'abord il n'a pas de but pratique et il semble que lors du juger, l'investissement des composantes disparates soit déchargé; ainsi s'expliquerait que les activités, "prédicat", se séparent du complexe du sujet en suivant une voie lâche.

On pourrait à partir d'ici entrer profondément dans l'analyse de l'acte de jugement, toutefois ceci nous écarte de notre thème.

Contentons-nous d'en retenir ceci: c'est l'intérêt originel à établir la situation de satisfaction qui a engendré, dans un cas, la réflexion reproductrice et dans l'autre le jugement comme moyen de parvenir de la situation de perception réellement donnée à celle qui est souhaitée. Le présupposé demeure donc ici que les processus $\Psi$ s'écoulent, non pas d'une façon inhibée, mais au contraire quand le Moi est actif. Le 
sens éminemment pratique de tout travail de pensée serait pourtant prouvé par làmême.

\section{8 - PENSER ET REALITE}

But et terme de tous les processus de pensée sont donc l'instauration d'un état d'identité, le passage d'une quantité d'investissement $(\mathrm{Q} \eta)$ provenant de l'extérieur dans un neurone investi à partir du Moi. Le penser qui reconnaît ou celle qui juge cherche une identité avec un investissement du corps, et la pensée qui reproduit en recherche une avec un investissement psychique (expérience propre). Le penser qui juge prépare le travail pour celui qui reproduit, en lui offrant des frayages achevés pour poursuivre la migration d'association.

$\mathrm{Si}$, après la conclusion de l'acte de penser, le signe de réalité s'ajoute à la perception, alors le jugement de réalité, la croyance sont obtenus et le but de tout le travail est atteint.

En ce qui concerne le juger, il est encore à remarquer que son fondement est manifestement la présence d'expériences propres du corps, de sensations et d'images de mouvement. Tant que celles-ci font défaut, la part transformante du complexe de perception demeure incomprise, c'est-à-dire qu'elle ne peut être reproduite, mais elle ne donne aucune direction pour d'autres cheminements de penser: c'est ainsi que par exemple -ce qui est important pour la suite- toutes les expériences sexuelles peuvent ne produire aucun effet, tant que l'individu ne connaît pas la sensation sexuelle, soit en général jusqu'au début de la puberté.

Le juger primaire semble présupposer une influence moindre du Moi investi que les actes de penser qui reproduisent de la poursuite. S'il s'agit ici d'une association par une superposition partielle à laquelle n'est appliquée aucune modification, il y a aussi des cas où le processus d'association du jugement s'accomplit avec la quantité pleine. La perception correspond à quelque chose comme un noyau d'objet + une image de mouvement. Tout en percevant $P$, on imite soi-même les mouvements, c'est-à-dire on innerve sa propre image de mouvement qui est éveillée suite à la coïncidence, et cela si fortement que le mouvement s'accomplit. Ainsi l'on peut parler d'une valeur d'imitation d'une perception. Ou bien la perception réveille l'image de souvenir de sa propre sensation de douleur, on ressent alors le déplaisir correspondant et l'on répète les mouvements de défense y afférents. C'est la valeur de compassion d'une perception. .

Dans ces deux cas nous pouvons voir le processus primaire dans son rapport au juger, et nous pouvons supposer que tout juger secondaire est advenu par la modération de ces processus purement associatifs. Le juger, plus tard moyen pour la reconnaissance de l'objet, et qui a peut-être une importance pratique, est donc à l'origine d'un processus associatif entre des investissements venant de l'extérieur et d'autres provenant du corps propre, une identification de $\Psi$ et d'informations internes ou d'investissements. II n'est peut-être pas injustifié de présumer qu'il représente en même temps une voie par laquelle des quantités (Q) venant de $\Phi$ peuvent être transportées et déchargées. Ce que nous nommons des choses sont des restes qui se soustraient au jugement.

L'exemple du jugement fournit d'abord un indice de la différence dans les quantités qui doit être statuée entre le penser et le processus primaire. 
Il est légitime de supposer que, lors du penser, un léger flux d'innervation motrice découle de $\Phi$, naturellement seulement si, dans le cours du processus, un neurone moteur ou un neurone clef a été innervé. Pourtant il serait injustifié de prendre cette décharge pour le processus lui-même dont elle n'est qu'un effet secondaire non intentionnel. Le processus de penser consiste dans l'investissement de neurones $\Psi$ avec altération de la contrainte du frayage par l'investissement latéral venant du Moi. Du point de vue mécanique, il est compréhensible que seulement une partie de la quantité $(Q \eta)$ peut suivre les frayages et que la grandeur de cette partie est régulée constamment par les investissements. Mais il est clair aussi que par là, suffisamment de quantité $(Q)$ est simultanément économisée pour rendre la reproduction un tant soit peu profitable. Dans l'autre cas, toute la quantité $(\mathrm{Q} \eta)$ qui à la fin est nécessaire à la décharge serait dépensée pendant le parcours aux points de sorties moteurs. Le processus secondaire est donc une répétition, à un niveau moindre, avec des quantités plus faibles, de l'écoulement originaire en $\Psi$.

Comment! Objectera-t-on, des quantités $(\mathrm{Q} \eta)$ encore plus petites que celles qui s'écoulent déjà dans les neurones $\Psi$ ! Comment fait-on pour ouvrir à de si petites quantités $(\mathrm{Q} \eta)$ les voies qui ne sont praticables que pour des quantités plus grandes que celles que $\Psi$ reçoit en règle générale? La seule réponse possible, c'est que ceci doit être une conséquence mécanique des investissements latéraux. II nous faut donc conclure aux relations suivantes: lors d'un investissement latéral, de petites quantités $(Q \eta)$ s'écoulent par des frayages là où par ailleurs seules de grandes quantités auraient trouvé passage. L'investissement latéral lie en quelque sorte une partie de la quantité $(Q \eta)$ s'écoulant par le neurone.

II faut par ailleurs que le penser satisfasse à une autre condition. Elle ne peut modifier essentiellement les frayages créés par les processus primaires, sinon elle falsifie les traces de la réalité. A cette condition satisfait la remarque que le frayage est vraisemblablement le résultat d'une unique grande quantité et que l'investissement, sur le moment très puissant, ne laisse néanmoins pas d'effet durable comparable. Les petites quantités $(\mathrm{Q})$ passant lors du penser, ne l'emportent en général pas sur les frayages.

Il est pourtant indubitable que le processus de penser laisse cependant derrière lui des traces durables, car (re)penser une seconde fois exige beaucoup moins de dépense que la première réflexion. Pour ne pas falsifier la réalité, il faut donc des traces particulières, des indices de processus de penser qui constituent une mémoire de penser, quelque chose à quoi nous ne pouvons pour l'instant donner forme. Nous verrons plus tard par quels moyens les traces des processus de penser sont séparées de celles de la réalité.

\section{9 - PROCESSUS PRIMAIRES - SOMMEIL ET REVE}

Maintenant surgit la question de savoir quelles sont les ressources quantitatives qui subviennent au processus primaire $\Psi$. Lors de l'événement de douleur, c'est manifestement la quantité $(Q)$ qui fait effraction en venant de l'extérieur, lors de l'affect c'est la quantité déliée par le frayage ; lors du processus secondaire de la pensée reproductrice une plus ou moins grande quantité $(\mathrm{Q \eta})$ venant du Moi peut manifestement être transférée sur le neurone c, qu'on peut désigner comme intérêt pour penser et qui est proportionnelle à l'intérêt pour l'affect, lorsqu'un tel intérêt a pu se produire. On peut seulement se demander s'il y a des processus $\Psi$ de nature primaire pour lesquels la quantité $(Q \eta)$ apportée depuis $\Phi$ suffit, ou bien si une 
contribution $\Psi$ (attention), qui seule rend possible un processus $\Psi$, s'ajoute automatiquement à l'investissement $\Phi$ d'une perception ? La question reste ouverte: ne pourrait-elle pas être tranchée par une adaptation spécifique à des faits psychologiques?

C'est un fait important que nous soyons en présence, quotidiennement durant le sommeil, de processus primaire $\Psi$ tels qu'ils ont été réprimés peu à peu au cours du développement biologique de $\Psi$. Second fait d'aussi grande portée: les mécanismes pathologiques que l'analyse la plus soigneuse découvre dans les psychonévroses ont la plus grande ressemblance avec les processus du rêve. Les conclusions les plus importantes résultent de cette comparaison qui demande à être élaborée ultérieurement.

Tout d'abord, le fait du sommeil doit être pris en compte dans la théorie. La condition essentielle du sommeil peut être reconnue clairement chez l'enfant. L'enfant dort aussi longtemps qu'aucun besoin ou aucune stimulation extérieure ne le tourmente (la faim ou le pipi froid). II s'endort avec la satisfaction (au sein). L'adulte dort aussi facilement post coenam et coïtum. La condition du sommeil est donc une diminution de la charge endogène dans le noyau $\Psi$ qui rend superflue la fonction secondaire. Dans le sommeil, l'individu se trouve dans l'état idéal de l'inertie, délivrée de la réserve de quantité $(Q \eta)$.

Cette réserve est au réveil accumulée dans le "Moi"; nous pouvons supposer que c'est la décharge du Moi qui est la condition du sommeil et qui le caractérise. Nous voyons tout de suite qu'est donnée ici la condition des processus primaires psychiques,

II n'est pas sûr que chez l'adulte le Moi se déleste complètement pendant le sommeil. De toute façon, il retire une infinité de ses investissements qui pourtant sont rétablis immédiatement et sans effort au réveil. Ceci ne contredit aucune de nos hypothèses, mais attire l'attention sur le fait qu'on doit supposer, entre des neurones bien liés, des courants qui, comme dans des vases communicants, concernent le niveau d'ensemble, bien que les hauteurs du niveau dans chaque neurone doivent seulement être proportionnelles mais non pas uniformes.

Des particularités du sommeil peuvent être déduites maintes choses qui ne se laissent pas deviner.

Le sommeil est caractérisé par une paralysie (de volonté) motrice. La volonté est la décharge de l'ensemble de la quantité $\Psi(Q \eta)$. Dans le sommeil le tonus spinal est partiellement relâché; il est vraisemblable que la décharge motrice $\Phi$ se manifeste par le tonus; d'autres innervations persistent avec leurs sources d'excitation.

Il est hautement intéressant que l'état de sommeil commence et est provoqué par la fermeture des organes des sens susceptibles d'être fermés. Les perceptions ne devant pas avoir lieu dans le sommeil, rien ne le perturbe plus que l'apparition d'impressions sensorielles, les investissements de $\Phi$ et se produisant en $\Psi$. Ceci semble indiquer que pendant la journée un investissement constant bien que déplaçable (attention) est envoyé vers les neurones du pallium qui reçoivent des perceptions à partir de $\Phi$; c'est ainsi que les processus primaires $\Psi$ peuvent très bien s'accomplir à l'aide de cette contribution $\Phi$. La question reste ouverte de savoir si les neurones du pallium ou aussi les neurones des noyaux adjacents sont eux-mêmes déjà pré-investis. Si $\Psi$ absorbe ces investissements du pallium, les perceptions se 
produisent en direction des neurones non investis, elles sont moindres, peut-être incapables de donner un signe de qualité à partir de perceptions. Comme nous l'avons présumé, avec l'évidement des neurones de perception $(\omega N)$ cesse alors aussi une innervation de décharge qui augmente l'attention. Nous arrivons ici à faire jonction avec l'énigme de l'acte d'hypnotiser. L'apparente inexcitabilité des organes sensoriels reposerait sur le retrait de l'investissement d'attention.

C'est donc par un mécanisme automatique -la contrepartie du mécanisme d'attention- que $\Psi$ exclut les impressions $\Phi$ tant qu'il est lui-même non investi.

Le plus remarquable est que dans le sommeil des processus $\Psi$ se déroulent les rêves, dont beaucoup de caractères restent incompris.

\section{0 - L'ANALYSE DES REVES}

Les rêves montrent tous les passages à l'état de veille et le mélange avec des processus $\Psi$ normaux, mais ce qui appartient en propre au rêve se laisse facilement extraire.

1. Les rêves manquent de décharge motrice comme, la plupart du temps, d'éléments moteurs On est, dans le rêve, paralysé. L'explication la plus aisée de ce caractère est la suspension de pré-investissement spinal par cessation de la décharge $\Phi$. L'excitation motrice ne peut dépasser la barrière quand les neurones ne sont pas investis. Dans d'autres états de rêve, le mouvement n'est pas exclu. Ceci n'est pas le caractère le plus essentiel du rêve.

2. Les nouages du rêve sont partiellement absurdes, partiellement débiles, ou bien même insensés, singulièrement fous.

Ce dernier caractère s'explique par ceci que, dans le rêve, la contrainte à l'association prévaut, comme c'est probablement le cas primairement dans la vie psychique. Il semble qu'il faille mettre en liaison deux investissements simultanément présents. J'ai collectionné des exemples comiques du règne de cette contrainte dans l'état de veille (par exemple, quelques provinciaux ayant assisté en France à la séance de la Chambre où un attentat eut lieu en ont tiré la conclusion que chaque fois qu'un député avait prononcé un bon discours, un coup de feu était tiré en signe d'applaudissement).

Les deux autres caractères qui sont à proprement parler identiques prouvent qu'une partie des expériences psychiques est oubliée. En effet, il est vrai que toutes les expériences biologiques qui inhibent ordinairement le processus primaire, et ceci en raison d'un manque d'investissement du Moi, sont oubliées. Le non-sens et l'illogisme du rêve est vraisemblablement attribuable au même caractère. II semble que les investissements $\Psi$ non absorbés s'égalisent en partie en prenant la direction des frayages les plus proches, en partie en prenant la direction des investissements voisins. Si le Moi se déchargeait complètement, alors le sommeil devrait être sans rêves.

3. Les représentations de rêve sont du genre hallucinatoire elles éveillent la conscience et trouvent crédit.

Ceci est le caractère le plus signifiant du sommeil. II se manifeste immédiatement lorsqu'il y a alternance de l'endormissement; on ouvre les yeux, on pense en mots. II y a plusieurs explications de la nature hallucinatoire des investissements du rêve. Tout d'abord on pourrait supposer que le courant à partir de $\Phi$ vers la motricité aurait 
empêché un investissement rétroactif des neurones $\Phi$ à partir de $\Psi$; quand ce courant cesse, $\Phi$ est investi de façon rétroactive et la condition de la qualité est remplie. J'objecte seulement la considération que les neurones $\Phi$, par leur noninvestissement, devraient être protégés de l'investissement à partir de $\Psi$, à l'instar de la motricité II est caractéristique du sommeil qu'il inverse ici tout le rapport: il suspend la décharge motrice de $\Psi$ et rend possible la décharge rétroactive vers $\Phi$. On pourrait être tenté de faire jouer là le rôle décisif au grand flux de décharge de l'éveil, de $\Phi$ vers la motricité. En second lieu, on pourrait recourir à la nature du processus primaire et indiquer que le souvenir d'une perception est toujours de I'hallucination et que c'est seulement l'inhibition du Moi qui nous a appris à ne jamais investir $P$ de telle façon qu'il puisse transférer rétroactivement sur $\Phi$.

Pour rendre cette supposition plus acceptable, on pourrait ici mentionner que la conduction $\Phi-\psi$ se passe dans tous les cas plus facilement que $\Psi-\Phi$, de sorte que même un investissement $\Psi$ d'un neurone qui excède de loin l'investissement de perception d'un même neurone n'a pas besoin de prendre la voie rétroactive. Cette explication se trouve encore confirmée par la circonstance que dans le rêve !a vivacité de l'hallucination est dans le même rapport avec l'importance, donc avec l'investissement quantitatif de la représentation concernée. Ceci renvoie au fait que c'est la quantité $Q$ qui conditionne l'hallucination. Si à l'état de veille une perception vient de $\Phi$, elle devient plus distincte par l'investissement $\Psi$ (intérêt), mais plus vivace, elle ne change pas son propre caractère quantitatif.

4. La finalité et le sens des rêves (tout au moins des rêves normaux) peuvent être déterminés avec certitude. Ce sont des accomplissements de vœux, donc des processus primaires d'après des événements de satisfaction et ils ne sont pas reconnus comme tels seulement parce que la déliaison de plaisir (reproduction de décharge de plaisir) y est faible, parce qu'ils se déroulent en général presque sans affect (sans déliaison motrice). Que ce soit leur nature, ceci est très facilement démontrable. C'est très précisément à partir de cela que je conclurais que l'investissement primaire de vœu était aussi de nature hallucinatoire.

5. Ce qui est remarquable, c'est la mauvaise mémoire que l'on en a et le peu de dégâts que les rêves causent par rapport aux autres processus primaires. Mais ceci s'explique facilement par le fait que les rêves prennent le plus souvent la voie des anciens frayages, donc ne provoquent pas de modification, et par le fait que les expériences $\Psi$ en sont mises à l'écart et que, en raison de la paralysie motrice, ils ne laissent pas de traces de décharge.

6. De plus, il est intéressant de constater que dans le rêve, la conscience fournit la qualité sans plus de perturbation que dans l'état de veille. Cela montre que la conscience ne colle pas au Moi, mais peut devenir un supplément à tous les processus $\Psi$. Elle nous met en garde d'identifier les processus primaires avec des processus inconscients.

Deux indications inestimables pour là suite ! $\mathrm{Si}$, le souvenir du rêve étant conservé, nous interrogeons avec insistance la conscience au sujet du contenu du rêve, il s'ensuit que la signification des rêves en tant qu'accomplissement de vœu est recouverte par une série de processus $\Psi$ qui se retrouvent tous dans les névroses et caractérisent leur nature morbide. 


\section{1 - LA CONSCIENCE DU REVE}

La conscience de la représentation du rêve est avant tout discontinue, ce n'est pas l'ensemble du déroulement associatif qui est devenu conscient, mais seulement différentes stations. Entre celles-ci se trouvent des moyens termes inconscients qu'on retrouve avec facilité à l'état de veille. Si nous cherchons la raison de ce saut, voici ce que nous trouvons; soit $A$ une représentation du rêve qui est devenue consciente et conduisant à $B$; mais au lieu de $B$, on trouve $C$ dans la conscience, et ceci parce que ce dernier se trouve sur la voie entre $B$ et un investissement $D$ simultanément présent.

II en résulte donc une dérivation par un investissement simultané, d'un genre différent, lui-même d'ailleurs non-conscient. $C$ s'est donc substitué à $B$, alors que $B$ correspond mieux à la liaison des pensées et à l'accomplissement de vœu. Par exemple, 0. a fait une injection de propyle à Irma, alors, je vois devant moi de façon très vive Triméthylamine, j'hallucine comme explication formelle: la pensée simultanément présente est la nature sexuelle de la maladie d'Irma. Entre cette pensée et le propyle il y a dans la chimie sexuelle une association dont j'ai discuté avec W. Fliess, lequel avait alors souligné la triméthylamine. Ceci devient conscient du fait de la pression ces deux côtés.

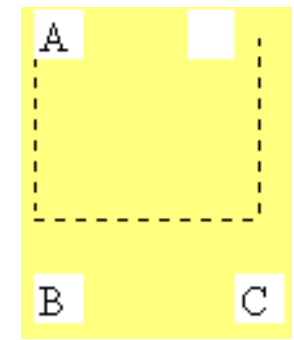

Il est fort énigmatique que ni le moyen terme (chimie sexuelle), ni la représentation dérivante (nature sexuelle de la maladie) ne deviennent, eux aussi, conscients, et ceci nécessite une explication. On pourrait penser que l'investissement de B ou de D n'est pas à lui seul assez intense pour forcer la voie jusqu'à l'hallucination rétroactive, alors que l'investissement bilatéral de C y arriverait. Seulement, dans l'exemple choisi, D (nature sexuelle) était certainement aussi intense que $A$ (injection de propyle), et le rejeton des deux, la formule chimique, était énormément vivace.

L'énigme de moyens-termes inconscients vaut également pour la pensée vigile où des situations ce genre arrivent tous les jours. Mais ce qui demeure caractéristique pour le rêve, c'est la facilité du déplacement de la quantité, et donc le remplacement de $\mathrm{B}$ par $\mathrm{C}$ préféré pour sa quantité.

II en va pareillement pour l'accomplissement de vœu dans le rêve. Ce n'est pas que le vœu devienne conscient et que son accomplissement soit alors halluciné, mais plutôt que ce dernier, le moyen-terme, reste à inférer. II a très certainement été traversé sans avoir pu se former qualitativement. Pourtant, on comprend l'impossibilité pour l'investissement de la représentation ce vœu d'être plus fort que le motif qui y pousse. Le déroulement psychique dans le rêve se produit donc selon la quantité $(\mathrm{Q})$; mais ce n'est pas la quantité $(\mathrm{Q})$ qui décide du devenir conscient. 
On peut peut-être encore tirer des processus du rêve que la conscience s'établit pendant un écoulement de quantité $(Q \eta)$, c'est-à-dire que ce n'est pas par un investissement constant qu'elle est éveillée. On doit en outre rencontrer la supposition qu'un courant intense de quantité n'est pas favorable à l'établissement de la conscience car cela entraîne le succès du mouvement, soit en quelque sorte un séjour plus tranquille de l'investissement. II est difficile, au milieu de ces déterminations contradictoires, de pénétrer jusqu'à ce qui fait la condition de la conscience. Aussi doit-on pour cela prendre en compte les relations par lesquelles la conscience s'établit dans le processus secondaire.

La singularité de la conscience du rêve mentionnée plus haut s'explique peut-être par le fait qu'un courant rétroactif de quantité $(Q \eta)$ vers $\Phi$ est incompatible avec un courant énergique vers les frayages d'association. D'autres conditions semblent valoir pour les processus $\Phi$ de la conscience.

25 septembre 95 


\section{IIe PARTIE - PSYCHOPATHOLOGIE}

La première partie de cette esquisse contenait ce qui pouvait se déduire quasiment à priori ce l'hypothèse fondamentale, modelée et corrigée selon différentes expériences effectives. Cette deuxième partie cherche à partir de l'analyse des processus pathologiques, d'autres déterminations du système qui repose sur l'hypothèse fondamentale; une troisième partie doit reconstruire à partir des deux précédentes, les caractères du déroulement psychique normal.

\section{PSYCHOPATHOLOGIE DE L'HYSTERIE}

\section{LA CONTRAINTE HYSTÉRIQUE}

Je commence par des choses qu'on rencontre dans l'hystérie, sans que pour autant elles lui soient nécessairement propres. -Chaque observateur de l'hystérie est d'abord frappé de ce que les hystéries sont soumises à une contrainte qui est exercée par des représentations surintenses. Par exemple, une représentation émerge, de façon particulièrement fréquente, dans la conscience sans que le déroulement puisse le justifier; ou bien le réveil de ces neurones s'accompagne de conséquences psychiques qu'on ne peut pas comprendre. A l'émergence de la représentation surintense sont liées certaines suites qu'il n'est possible ni de réprimer ni de comprendre: des déliaisons d'affect, des innervations motrices, des empêchements. A l'individu n'échappe nullement l'aspect frappant de cet état de fait. Des représentations surintenses, il en existe aussi normalement. Elles confèrent au Moi sa singularité. Nous ne nous étonnons pas, lorsque nous connaissons leur développement génétique (éducation, expériences) et leurs motifs. Nous sommes habitués à considérer de telles représentations surintenses comme le résultat de motifs importants et justifiés. Les représentations surintenses dans l'hystérie nous frappent par contre par leur singularité, ce sont ces représentations qui chez !es autres sont sans conséquences, et dont nous ne comprenons en rien le prix. Elles nous apparaissent comme des parvenues, des usurpatrices, donc ridicules.

La contrainte hystérique est donc 1 ) incompréhensible; 2 ) insoluble par le travail de penser; 3 ) incongrue dans son montage.

II y a une contrainte névrotique simple que l'on peut mettre en opposition avec la contrainte hystérique, par exemple: un homme est tombé d'une voiture, s'est trouvé en danger et depuis, il ne peut plus prendre une voiture. Cette contrainte est 1) compréhensible, car nous connaissons son origine ; 3) pas incongrue car l'association avec le danger justifie le nouage (Verknüpfung) de l'usage de la voiture avec la peur. Mais elle aussi n'est pas soluble par le travail de penser. On ne peut pas considérer ce dernier caractère comme entièrement pathologique, nos idées normales surintenses sont aussi souvent insolubles. On tiendrait la contrainte névrotique pour nullement pathologique, si l'expérience ne montrait qu'une telle contrainte chez un homme en bonne santé ne persiste que peu de temps après ce qui l'a occasionné, puis se brise avec le temps. La persistance de la contrainte est donc pathologique et renvoie à une névrose simple.

De nos analyses, il résulte que la contrainte hystérique est immédiatement résolue lorsqu'elle est éclaircie (rendue compréhensible). Au fond, ces deux caractères n'en font qu'un. Dans l'analyse, on apprend aussi par quel processus l'apparence d'absurdité et d'incongruité s'est établie. Le résultat de l'analyse est en termes généraux le suivant: Avant l'analyse, $A$ est une représentation surintense qui exerce trop souvent une poussée vers la conscience et provoque chaque fois des 
pleurs. L'individu ne sait pas pourquoi $A$ le fait pleurer, il trouve cela absurde, mais il ne peut pas l'empêcher.

Après l'analyse on a trouvé qu'il y a une représentation $B$, laquelle provoque à juste titre des pleurs et qui, à juste titre, se répète souvent tant que ne s'est pas accompli chez l'individu un certain travail psychique compliqué à son endroit. L'effet de $B$ n'est pas absurde, II est compréhensible à l'individu et peut même être combattu par lui. $B$ se trouve vis-à-vis de $A$ dans un rapport déterminé. En effet, il y a eu un événement qui consistait en $B+A$. A était une circonstance accessoire, $B$ était propre à produire cet effet qui demeure. Dès lors la reproduction de cet incident a pris forme dans le souvenir comme si A avait pris la place de B. A est devenu le substitut, le symbole de $B$. D'où le caractère incongru: $A$ est accompagné de suites dont il ne semble pas être digne, qui ne lui vont pas.

Les formations de symboles se produisent aussi de façon normale. Le soldat se sacrifie pour un chiffon bariolé sur un bout de bois, parce que ce chiffon est devenu le symbole de la patrie et personne ne trouve cela névrotique.

Mais le symbole hystérique se comporte autrement. Le chevalier qui se bat pour le gant de la Dame sait premièrement que le gant doit sa signification à la Dame et deuxièmement il n'est nullement empêché par la vénération de ce gant de penser à la Dame et de la servir par ailleurs. L'hystérique que $A$ fait pleurer ne sait absolument pas qu'il pleure à cause de l'association $A$ - B, et $B$ lui-même ne joue dans sa vie psychique strictement aucun rôle. Le symbole s'est complètement substitué à la chose (das Ding).

Cette affirmation est exacte au sens le plus rigoureux. On se persuade de ce que lors de tous les réveils qui, de l'extérieur et à partir de l'association, devraient à vrai dire investir $B$, $A$ pénètre à sa place dans la conscience. En effet, de la nature des occasions qui -de façon étrange- éveillent $A$, on peut déduire la nature de $B$.

Résumons l'état des choses : A a un caractère de contrainte, B est refoulé (du moins hors de la conscience).

L'analyse a révélé ce résultat surprenant; à chaque contrainte correspond un refoulement, à chaque intrusion démesurée dans la conscience correspond une amnésie.

Le terme "surintense" renvoie à des caractères quantitatifs; on n'est pas loin de supposer que le refoulement a le sens quantitatif d'un dénudement de quantité ( $Q$ ) et que la somme des deux équivaut à la normale. Ce n'est donc que la répartition qui a changé. Quelque chose a été ajouté à $A$ qui a été soustrait à $B$, le processus pathologique est celui d'un déplacement comme nous avons appris à le connaître dans le rêve donc un processus primaire $\Psi$.

\section{2 - LA CONSTITUTION DE LA CONTRAINTE HYSTÉRIQUE}

Maintenant se lèvent plusieurs questions qui ne sont pas vides de contenu. Dans quelles conditions en vient-on à une telle formation pathologique de symboles ou (d' autre part) au refoulement ? Quelle est ici la force motrice? Dans quel état se trouvent les neurones de la représentation surintense et ceux de la représentation refoulée?

II n'y aurait là rien d'autre à deviner et rien de plus à construire si l'expérience clinique n'enseignait pas deux états de fait. Le refoulement concerne toujours des 
représentations qui, premièrement, éveillent un affect pénible (déplaisir) pour le Moi, deuxièmement sont issues de la vie sexuelle.

On peut déjà supputer que c'est cet affect de déplaisir qui fait aboutir le refoulement. En effet, nous avons déjà admis une défense primaire qui consiste en ceci que le courant de penser s'inverse dès qu'il bute sur un neurone dont l'investissement délie du déplaisir.

Ceci a trouvé une justification dans deux expériences: premièrement, cet investissement de neurones n'est assurément pas celui qui est recherché au moment où le processus de penser avait à l'origine pour but l'instauration de la situation de satisfaction $\Psi$, deuxièmement, lorsque prend fin de façon réflexe un événement de douleur, la perception hostile est remplacée par une autre.

On peut toutefois se persuader plus directement du rôle de l'affect de défense. Si l'on cherche l'état dans lequel se trouve la représentation (Vorstellung) refoulée $B$, on découvre alors que celle-ci est facile à trouver et à porter à la conscience. Ceci est une surprise; en effet on aurait pu penser que B était effectivement oublié, qu'aucune trace de souvenir de B n'était restée en $\Psi$. Non, B est une image de souvenir comme une autre, elle n'est pas éteinte. Mais si, comme d'ordinaire, B est un complexe d'investissement, une forte et peu commune résistance, difficile à éliminer, s'élève contre le travail de penser avec $B$. On peut sans difficulté voir dans cette résistance contre $B$ la mesure de la contrainte qu'exerce $A$, et on peut croire qu'on voit ici à nouveau au travail la force qui, en son temps, a refoulé $B$. Nous apprenons en même temps autre chose. Jusqu'ici, en effet, nous savions seulement que $B$ ne peut pas devenir conscient; quant au comportement de B à l'égard de l'investissement de penser, rien n'était connu. Maintenant nous apprenons que chaque fois que le penser s'occupe de $B$, la résistance se tourne contre elle, même si cela a déjà été rendu partiellement conscient. Au lieu de "exclu de la conscience" on peut donc écrire: exclu du processus de penser.

II s'agit donc d'un processus de défense provenant du Moi investi lequel a comme conséquence le refoulement hystérique et par suite la contrainte hystérique. En ceci le processus semble se séparer des processus primaires $\Psi$.

\section{3 - LA DÉFENSE PATHOLOGIQUE}

Nous sommes cependant très éloignés d'une solution. La réussite du refoulement hystérique diffère, comme nous le savons, dans une très large mesure, de celle de la défense normale que nous connaissons avec exactitude. En général nous évitons de penser à ce qui n'éveille que du déplaisir, et nous le faisons en dirigeant nos pensées vers autre chose. Si toutefois nous arrivons ensuite à ce que la représentation $B$ incompatible émerge rarement dans notre conscience, parce que nous l'avons maintenue isolée le plus possible, nous ne réussissons pourtant jamais à oublier $\mathrm{B}$ au point que nous ne puissions y être rappelés par une nouvelle perception. Un tel éveil ne peut pas être empêché même dans le cas de l'hystérie, la différence ne consiste qu'en ceci qu'au lieu de B c'est toujours A qui devient conscient, donc qui est investi. C'est donc une formation de symboles d'une si grande fixité qui constitue cette réalisation allant au-delà de la défense normale.

L'explication on la plus immédiate de ce plus-de- travail serait qu'il faille incriminer la plus grande intensité de l'affect de défense. L'expérience toutefois montre que les souvenirs les plus pénibles, qui doivent nécessairement éveiller le plus grand déplaisir (souvenirs de remords relatifs à de mauvaises actions), ne peuvent pas être 
refoulés ni être remplacés par des symboles. L'existence de la deuxième condition pour la défense pathologique -la sexualité- indique que l'explication est à chercher ailleurs.

II est tout à fait impossible d'admettre que les affects sexuels pénibles dépassent à ce point en intensité tous les autres affects de déplaisir. Ce doit être un autre caractère de la représentation sexuelle qui peut expliquer que seules des représentations sexuelles sont soumises au refoulement.

Ajoutons ici encore une remarque. Le refoulement hystérique survient manifestement grâce à l'aide de la formation de symboles, du déplacement sur d'autres neurones. On pourrait alors penser que l'énigme ne repose que sur le mécanisme de ce déplacement, qu'il n'y aurait rien à expliquer quant au refoulement lui-même. Pourtant l'analyse, par exemple, de la névrose obsessionnelle nous apprendra que là a lieu du refoulement sans formation de symboles et même que refoulement et substitution s'y disjoignent quant au temps. Ainsi le processus du refoulement demeure le noyau de l'énigme.

\section{LE $\pi \rho \omega \tau$ Tov $\psi \varepsilon 1 \delta 0 \sigma^{1}$ DE L'HYSTÉRIQUE}

Nous avons appris que la contrainte hystérique provient d'une forme singulière de mouvement de quantité (formation de symboles), qui est vraisemblablement un processus primaire, puisqu'il se laisse facilement démontrer dans le rêve; nous savons que la force motrice de ce processus est la défense du Moi qui. accomplit: toutefois ici plus que sa fonction normale. Le fait que lors du processus du Moi, s'établissent des conséquences dont nous n'avons l'habitude que lors des processus primaires, requiert une explication II faut s'attendre ici à des conditions psychiques particulières. Du point de vue clinique, nous savons que tout ceci ne se passe que dans le domaine sexuel; peut-être avons-nous donc à expliquer la condition psychique particulière à partir de caractères naturels de la sexualité.

Or, dans le domaine sexuel, il y a précisément une constellation psychique particulière qui pourrait servir notre intention. Nous allons l'exposer à l'aide d'un exemple, qui nous est connu par l'expérience.

Emma subit aujourd'hui une contrainte : elle ne peut pas aller seule dans un magasin. Pour expliquer ceci, elle évoque un souvenir de sa douzième année (peu après sa puberté!. Elle alla acheter quelque chose dans un magasin, vit rire ensemble les deux commis, dont l'un resta dans son souvenir, et elle s'enfuit frappée d'une sorte d'affect d'effroi De là la pensée que les deux ont ri de sa robe et que l'un lui a plu sexuellement. La relation de ces fragments, ainsi que l'effet de l'événement, sont incompréhensibles.

Si elle a ressenti du déplaisir parce qu'on se moquait d'elle à cause de sa robe, cette impression aurait dû se rectifier depuis longtemps, depuis qu'elle est habillée comme une dame; de même, le fait d'aller seule ou accompagnée dans le magasin ne change rien à son vêtement. Qu'elle n'ait pas directement besoin de protection s'explique par le fait que, comme dans l'agoraphobie, l'accompagnement d'un petit enfant suffit pour lui apporter la sécurité. Ce qui ne colle pas du tout avec le reste, c'est qu'un des deux commis lui ait plu; un accompagnement n'y changerait rien non

\footnotetext{
${ }^{1}$ Expression employée par Aristote in "Premiers Analytiques" L..II chap.. 18, 66a, 16 ("Une proposition fausse est le résultat d'une erreur qui précède"). Heinrich Hertz utilise précisément cette expression en 1894 (ce. Olla Anderson, La préhistoire de la psychanalyse, 1962). Cf. S.E. 1, p. 352
} 
plus. Les souvenirs éveillés n'expliquent donc ni la contrainte ni la détermination du symptôme.

Une recherche supplémentaire découvre alors un deuxième souvenir, elle conteste qu'elle l'ait eu au moment de la scène I, ce qui n'est en effet démontré par rien. A l'âge de huit ans, elle alla deux fois seule dans le magasin d'un petit vieux pour acheter des sucreries. Le brave homme la pinça à travers ses vêtements aux organes génitaux. Malgré la première expérience, elle y retourna une deuxième fois. Après elle demeura absente. Elle se fait maintenant des reproches d'y être retournée une deuxième fois, comme si par là elle avait voulu provoquer l'attention En effet, un état de "mauvaise et pesante conscience" doit être rapporté à cet événement.

Nous comprenons dès lors la scène I (commis) si nous ajoutons la scène II (petit vieux). Nous n'avons besoin que d'une liaison associative entre les deux. Elle indique elle-même que celle-ci serait donnée par le rire. Le rire des commis l'aurait fait penser au ricanement avec lequel le petit vieux avait accompagné son attentat. Alors le processus se laisse reconstruire comme suit: dans le magasin, les deux commis rient, ce rire éveille (inconsciemment) le souvenir du petit vieux. La situation présente en effet une autre analogie; elle est de nouveau seule dans le magasin. Avec le petit vieux est remémoré le pincement à travers les vêtements, mais depuis elle est devenue pubère. Le souvenir éveille alors ce qu'à l'époque il ne pouvait certainement pas éveiller, une déliaison sexuelle qui se transpose en angoisse. De par cette angoisse, elle craint que les commis ne puissent répéter l'attentat, elle s'enfuit.

Il est tout à fait assuré qu'ici deux sortes de processus sont intriquées, que le souvenir de la scène II (petit vieux) se produisit dans un autre état que le précédent. Le déroulement peut être figuré comme suit :

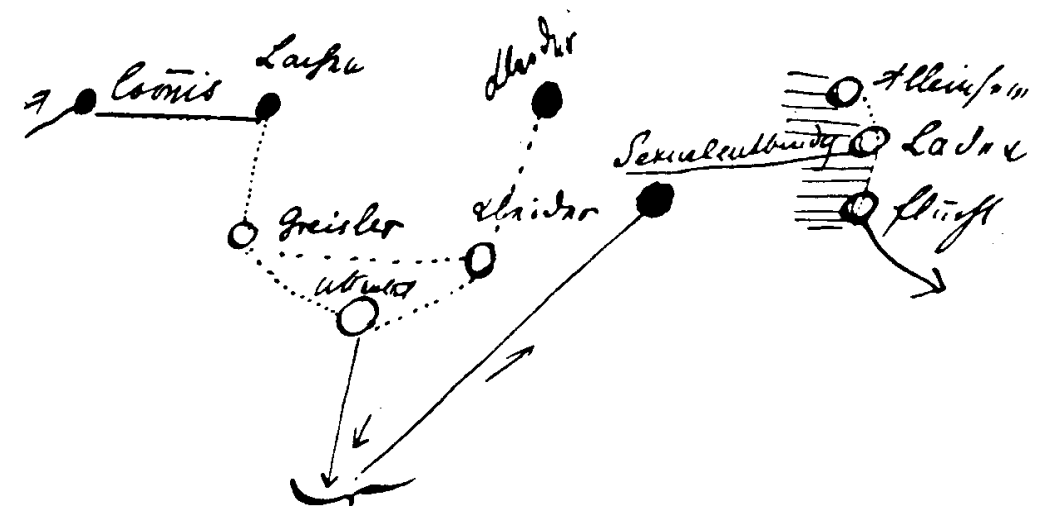

Dans ce schéma, les représentations $(R)$ figurées en noir sont des perceptions qui sont aussi remémorées. Le fait que la déliaison sexuelle vienne à la conscience prouve l'idée -sans cela incompréhensible- que le commis qui a ri lui a plu. La conclusion de ne pas rester seule dans le magasin en raison du danger d'attentat, est tout à fait correctement construite, si l'on prend en considération tous les éléments du processus d'association. Mais du processus figuré dans la partie inférieure du schéma, rien n'est venu à la conscience si ce n'est l'élément "vêtements" et le penser travaillant avec la conscience a formé à partir du matériel présent (commis, rire, vêtements, sensation sexuelle) deux faux nouages : c'est-àdire qu'on a ri d'elle à cause de ses vêtements et qu'elle a été attirée sexuellement par l'un des commis. 
Tout le complexe (lignes brisées) est représenté dans la conscience par une représentation "vêtements", manifestement la plus anodine. II s'est produit ici un refoulement avec formation de symboles. II fait que la conclusion -le symptôme- est alors construit tout à fait correctement de telle sorte que le symbole n'y joue aucun rôle, est à vrai dire une particularité du cas.

On pourrait dire qu'il est tout à fait habituel qu'une association passe par des moyens termes inconscients jusqu'à ce qu'elle rencontre un moyen terme conscient, comme cela se passe ici. Vraisemblablement pénètre alors dans la conscience le terme qui éveille un intérêt particulier. Dans notre exemple toutefois ceci est précisément remarquable que ne pénètre dans la conscience non pas le terme qui éveille un intérêt (attentat) mais un autre en tant que symbole (vêtements). Si l'on se pose la question de savoir quelle pourrait être la cause de ce processus pathologique inséré, alors on ne trouve qu'une seule, la déliaison sexuelle, qui est aussi attestée dans la conscience. Celle-ci est nouée au souvenir de l'attentat, mais il est tout à fait remarquable qu'elle n'était pas nouée à l'attentat lorsqu'il a été vécu. Nous nous trouvons ici devant le cas où un souvenir éveille un affect qu'il n'avait pas éveillé en tant qu'événement parce qu'entre temps le changement de la puberté a rendu possible une autre compréhension de ce qui est remémoré.

Ce cas est vraiment typique du refoulement dans l'hystérie. Dans tous les cas il se trouve qu'un souvenir est refoulé qui n'est devenu un trauma qu'après-coup. La cause de cet état de chose est le retard de la puberté par rapport aux autres aspects du développement de l'individu

\section{LES CONDITIONS DU $\pi \rho \omega \tau 0 v \psi \varepsilon \varepsilon v \delta 0 \sigma$}

Bien que, dans la vie psychique, il n'arrive habituellement pas qu'un souvenir éveille un affect qu'il n'a pas amené avec lui en tant qu'événement, ceci est quand même quelque chose de tout à fait habituel pour la représentation sexuelle, précisément parce que le retard de la puberté est un caractère général de l'organisation. Tout adolescent a des traces de souvenir qui ne peuvent être comprises que lors de l'apparition de ses propres sensations sexuelles, chacun devrait donc porter en lui le germe de l'hystérie. Manifestement il faudrait encore d'autres facteurs, agissant de concert, si cette nécessité générale devait se limiter au petit nombre de personnes qui deviennent effectivement hystériques.

En effet, l'analyse indique que ce qui perturbe dans un trauma sexuel est manifestement la déliaison d'affect, et l'expérience apprend à connaître les hystériques comme des personnes dont d'une part on sait qu'elles sont devenues sexuellement excitables précocement (masturbation) par stimulation mécanique et émotionnelle et dans !a disposition desquelles, d'autre part, on peut supposer une déliaison sexuelle précoce. Mais un commencement précoce de la déliaison sexuelle et une déliaison sexuelle précocement plus forte sont manifestement équivalents. Cette fonction se réduit à un facteur quantitatif.

Mais en quoi devrait donc consister la signification de la précocité dans la déliaison sexuelle? La précocité pèse ici de tout son poids, car on ne peut pas soutenir que la déliaison sexuelle fournisse toujours le motif du refoulement ; ceci ferait à nouveau du refoulement un processus de fréquence normale. 


\section{LA PERTURBATION DU PENSER PAR L'AFFECT.}

Nous n'avons pu rejeter le fait que la perturbation du processus psychique normal avait deux conditions: 1) que !a déliaison sexuelle s'est nouée à un souvenir au lieu de se nouer à un événement, 2) que la déliaison sexuelle a eu lieu précocement.

Par ces deux addenda une perturbation devrait être causée qui dépasse la mesure normale, mais qui est aussi préfigurée dans le normal.

On fait quotidiennement l'expérience que le développement d'affect inhibe le développement normal du penser et cela de différentes façons. Premièrement, beaucoup de voies du penser qui autrement seraient prises en compte sont oubliées, donc à l'instar de ce qui se passe dans le rêve; il m'est par exemple arrivé d'oublier dans l'excitation un gros souci de me servir du téléphone installé chez moi depuis peu de temps. La voie récemment tracée succombe dans l'état d'affect. Le frayage c'est-à-dire l'ancienneté a pris le dessus. Avec cet oubli disparaît la sélection, l'appropriation à un but et la logique du déroulement, tout comme dans le rêve. Deuxièmement: sans cet oubli, des voies sont empruntées qui sinon, sont évitées, surtout des voies servant la décharge, comme par exemple des actions dans l'affect. Finalement, le processus d'affect est proche du processus primaire non-inhibé.

De ceci maintes conclusions sont à tirer. Premièrement: lors de la déliaison d'affect, la représentation qui délie est elle-même renforcée; deuxièmement, la tâche principale du Moi investi consiste dans la prévention de l'affect et dans la réduction des anciens frayages d'affect. On ne peut se représenter le rapport de ces faits que de la façon suivante. A l'origine un investissement de perception, en tant qu'héritier d'un événement de douleur, a délié du déplaisir, il s'est renforcé par la quantité déliée et a procédé maintenant à la décharge sur les voies d'écoulement en partie défrayées. Après qu'un Moi investi a été construit, "l'attention" dirigée contre de nouveaux investissements de perception s'est développée d'une manière qu'on connaît, attention qui suivait alors le déroulement à partir de $P$ au moyen d'investissements latéraux. En ceci la déliaison ce déplaisir a été quantitativement limitée, et son commencement était pour le Moi précisément un signal disant: procédez à une défense normale; ainsi la production trop aisée de nouveaux événements de douleur avec leurs frayages a été empêchée. En effet, plus la déliaison du déplaisir est forte, plus est difficile la tâche du Moi qui, avec ses investissements latéraux, ne peut fournir un contrepoids aux quantités jusqu'à une certaine limite et qui doit donc admettre un écoulement primaire

De surcroît - plus la quantité tendant à l'écoulement est grande, plus est difficile le travail de penser pour le Mol, travail qui consiste, selon toutes les indications, en un glissement à titre d'essai de petites quantités $(\mathrm{Q} \eta)$. L'acte de "réfléchir" est une activité du Moi qui exige du temps, qui ne peut pas avoir lieu quand le niveau de l'affect met en jeu de grandes quantités $(Q \eta)$. De là la précipitation et un choix des voies dans l'affect, ressemblant à celui qui a lieu dans le processus primaire.

Pour le Moi il s'agit donc de ne pas admettre de déliaison d'affect parce qu'il admettrait alors un processus primaire. Son meilleur instrument à cet égard est le mécanisme de l'attention. Si un investissement déliant du déplaisir pouvait se soustraire à ce dernier, le Moi serait en retard par rapport à cet investissement. C'est précisément ce cas qui se présente avec le proton pseudo hystérique. L'attention est réglée sur les perceptions qui, sinon, donnent lieu à la déliaison de déplaisir. Ici ce n'est pas une perception mais une trace de souvenir qui délie du déplaisir de façon 
insoupçonnée, et le Moi n'en est informé que trop tard; il a admis un processus primaire parce qu'il n'en attendait pas.

Toutefois il arrive aussi par ailleurs que ces souvenirs délient du déplaisir. Certes, pour des souvenirs frais c'est tout à fait normalement le cas. D'abord, quand le trauma (événement de douleur) advient les tout premiers traumas échappent tout à fait au Moi - à une époque où il y a déjà un Moi, une déliaison de déplaisir se produit mais en même temps le Moi est également occupé à créer des investissements latéraux. Si l'investissement de la trace de souvenir se répète le déplaisir se répète aussi, mais les ravages du Moi sont déjà présents; l'expérience montre que !a deuxième fois la déliaison est moins :importante, jusqu'à ce qu'elle s'amenuise au cours de répétitions ultérieures pour aboutir à l'intensité d'un signal agréable au Moi. II s'agit donc seulement de ceci : lors de la première déliaison de déplaisir l'inhibition du Moi ne fait pas défaut, le processus ne se déroule pas comme un événement d'affect primaire posthume, et c'est précisément cela qui s'accomplit quand, comme dans le cas du proton pseudo hystérique, c'est avant tout le souvenir qui donne lieu à la déliaison de déplaisir.

Une des conditions précédemment mentionnées que nous livre l'expérience clinique verrait sa signification correctement appréciée. Le retard de la puberté rend possible des processus primaires posthumes. 